\title{
The unified model description of order-disorder and displacive structural phase transitions
}

\author{
S.Stamenković \\ Institute of Nuclear Sciences, Laboratory for Theoretical Physics and \\ Condensed Matter Physics, Belgrade, P.O.Box 522, Yugoslavia
}

Received July 7, 1998

\begin{abstract}
A series of co-authors' studies [1-7] devoted to the unified model description of structural phase transitions (SPT) in ferroelectrics and related materials are reviewed and partly innovated.
\end{abstract}

Starting from a general Hamiltonian of pair-coupled anharmonic (quartic) oscillators, together with the concept of local normal coordinates, a unified model description of both order-disorder and displacive types of SPT-systems is proposed. Within the framework of the standard variational procedure, a hybridized pseudospin-phonon Hamiltonian is formulated by introducing variables corresponding to phonon, magnon-like(flipping) and nonlinear(domain-wall-like) displacements of atoms participating in SPT. This is achieved by representing the cooperative atomic motion onto several quasiequilibrium positions (in the simplest case, two) as slow tunnelling displacement (decomposed into magnon-like and soliton-like deviations), in addition to comparatively fast phonon oscillations around inhomogeneous momentary rest positions, in turn induced by domain-wall-like (soliton) excitations.

The qualitative and quantitative analyses show that SPT (of the first or second order) can be either of a displacive (governed by a phonon soft mode), order-disorder (governed by a tunnelling-magnon-like soft mode) or of a mixed type, depending on both the coupling energy between atoms and their zero-point vibrational energy. In the critical temperature region, the domain-wall-like excitations bring on the formation of microdomains (precursor clusters of the ordered phase) which induce SPT of the Ising type universality class. The incomplete softening of the phonon or pseudomagnon mode occurs and a central peak due to slow cluster relaxation appears in the spectral density of excitations.

Key words: structural phase transitions, order-disorder,displacive transition

PACS: $63.70 .+h, 77.80 . B h, 64.60 .-i, 64.60 . C n$ 


\section{Introduction}

Among numerous attempts to develop a unified microscopic theory of both displacive and order-disorder structural phase transitions (SPTs), on the occasion of the 60th anniversary of Prof. Stasyuk's birthday, our modest contribution [1-7] to the same problem in ferroelectric phase transitions is reviewed and partly innovated. To expose as clear as possible the basic proposals for a unified description of SPT-systems and to better comprehend this peculiar problem in connection with its prospective development, we shall try to keep mostly to the original studies of the co-authors' group, with reference to other similar approaches cited in the co-authors' publications. Accordingly, to acquire a rather contemporary unified description of SPT (i.e. by novelizing the previous, original ones) it is convenient to employ the representation of local normal coordinates (LNCs) involving cooperative displacements of all the atoms in a primitive cell participating in the given critical (soft) vibrational mode. The LNCs are chosen so that they take into account the symmetry properties of the system, in order to be transformed by an irreducible representation of the point group of the primitive cell. Since the corresponding order parameter of the system is transformed by the irreducible representation of the space group, such "conjunctive" coordinates can be considered as the appropriate basis for describing SPT in the case when a single channel transition (corresponding to a single-relevant irreducible representation) takes place in the system we deal with throughout this review. With this substantial concept in mind, the grounds for the unified description of SPT become physically transparent when one appreciates the natural generalization of the traditional concept of atomic (including cooperative-like, represented by LNC) equilibrium states. In the next step the time dependent LNC attributed to the cooperative atomic motion within the effective anharmonic multi-well potential is decomposed into a "slow" tunnelling displacement (being a momentary rest position, it consists of flippingmagnon-like and domain-wall-soliton-like deviations) and a comparatively "fast" superimposed deviation of the phonon type around the inhomogeneous cluster induced by quasi-equilibrium positions (in the simplest case, two) inside the effective one-particle potential. Thus, the ordering process is characterized by two order parameters - one reflecting the degree of the local precursor order (the average population of equilibrium positions $\sigma^{z}$ ) and the other controlling the degree of the long-range order (the average atomic displacement $\eta$ ).

The underlying approach is developed by incorporating the already adopted LNC and the corresponding canonical momentum into the familiar model Hamiltonian expressed in the form of effective pair-coupled anharmonic (in the simplest case, quartic) oscillators. The further procedure is completed by a variational adaptation of the microscopic model Hamiltonian (in the reduced phase space of coordinates and momenta) in various hybridized forms of phonon - tunnelling (pseudospin) - domain-wall (soliton) types separated in corresponding variables. On the basis of a self-consistent phonon and a molecular-field approximations (namely, a combination of an independent-mode and independent-site evaluation schemes), a 
complete set of coupled self-consistent equations is obtained which enables one to estimate or to calculate (mainly numerically) all the physical (collective or local) quantities of the SPT-system (in the first place, both order parameters included). The qualitative and quantitative analyses of such a system of equations show that the SPT (of the first or second order) can be of a displacive (governed by a phonon soft mode), order-disorder (governed by a tunnelling - magnon-like soft mode) or a mixed type - depending predominantly on the reduced lattice coupling strength and, in a lesser degree, on the ratio of the zero-point vibrational energy to the height of the single-particle potential barrier. The possibility for phase transitions of the both types occuring at zero temperature (quantum limits) is also outlined. In the case of a strongly anharmonic system the quantum limit of the displacive type is examined in both, the ordered $\left(\sigma^{z}=1\right)$ and disordered $\left(\sigma^{z}=0\right)$ lattices.

Finally, it is analytically demonstrated that with the onset of criticality in all, order-disorder, displacive, as well as in mixed types of structural phase transitions, the domain-wall (soliton) excitations bring on the formation of microdomains (precursor clusters of the ordered phase) which induce a phase transition of the Ising universality class. The incomplete softening of the phonon or/and pseudomagnon mode occurs and a central peak due to the slow cluster relaxation appears in the energy spectrum of excitations.

\section{Preliminaries}

\subsection{The elements of lattice dynamics at a structural transition}

In agreement with the soft mode concept put forward independently by several scholars in this field of solid state physics*, a lattice undergoing transition is not stable in the harmonic approximation (it has a purely imaginary frequency for the optical, i.e. polar mode) owing to the compensation of long-range attractive and short-range repulsive forces. In that case in the symmetric phase the anharmonic interaction, even if it is small, is necessary for the lattice vibrations to be stable. Therefore, in a consistent dynamical theory of SPT one must take into account the anharmonic interaction from the very beginning (already in the zero approximation), which requires the application of the methods of statistical mechanics, and depart from the simple mechanical approach adopted in the Born-Karman theory. Meantime, a self-consistent phonon theory was developed ( mainly in connection with the study of quantum helium crystals) which was also applied to the description of structural transitions.

As it is well known, unlike the customary self-consistent (molecular) field approximation, in the self-consistent phonon theory account is taken of fluctuations of the order parameter, which play an important role in the second-order phase transitions. It should be pointed out that the self-consistent phonon theory makes the renormalised (due to fluctuations) Landau expansion for the free energy possible and allows a self-consistent determination of the range of applicability of this

${ }^{*}$ For a historical outline, for example, see [4] in [7]. 
method. The self-consistent phonon theory itself is generally considered for an arbitrary lattice (e.g., in $[7,8]$ ), where various theoretical applications to the concrete SPT model (we will also deal with them hereafter) are surveyed in detail. Such a theory is also adjusted to describe order-disorder SPT or transitions of the mixed type which differ from the displacive ones only by a significantly higher degree of unharmonicity and the related relaxational nature of critical-mode excitations (see Subsections 3.5 and 3.7).

To highlight the basic physical consequences including the main quantitative features of the unified description of the proposed SPT, it is sufficient to use only the first-order self-consistent phonon approximation (also named the pseudoharmonic or renormalised phonon approximation), favoured, in addition, by its simplicity. It is worthwhile to note that in the studies of strongly anharmonic SPT-systems the so-called improved first-order self-consistent phonon (namely, the improved pseudoharmonic) approximation is often used. Likewise, within the framework of such an approximation the interaction of pseudoharmonic phonons is then described through the self-energy operator which determines the frequency shift and the damping of self-consistent phonons. The self-energy operator itself is then calculated self-consistently in the simplest approximation with taking proper account of the uncorrelated propagation of renormalised (in the intermediate state) phonons $^{* *}$.

\subsection{The microscopic model description of structural phase transitions}

It is well established that in spite of the self-consistent phonon theory capable of accommodating the exact relevant expressions, it is not always possible to carry out concrete or explicit calculations with their aid. Hence, for a model description including a proper interpretation of experimental data, it is instructive to single out from a complete microscopic picture of the phenomenon, only its most essential features rendered into a simple physical model. The corresponding well conceived models permit one to clarify the scope of computational methods and the limits of their applicability. In the case of SPT only low-lying soft modes (responsible for the phase transition) and those phonon modes permitted (by the system symmetry) to interact with them are important. Therefore, in a model description it is physically quite acceptable to consider only a small number of normal lattice vibrations and their anharmonic interactions. As most SPTs are accompanied by a lattice deformation, in discussing real systems in terms of LNCs, of particular interest is to take into account the coupling of the critical mode with the relevant acoustic mode.

\subsubsection{The concept of the local normal coordinate}

The inherent ingredients of any physically founded approach in describing SPTsystems and searching for their unified description certainly are the symmetry

\footnotetext{
${ }^{* *}$ The sizable survey of various current self-consistent phonon approximations with their findings
} included is given in $[7,8]$ and Refs. therein. 
properties apparently associated with crystal structures of these compounds. Thus, the symmetry requirements imposed on SPT-systems (locally and on the whole crystal) have to be taken into consideration and built in properly into the adopted model, namely, into the Hamiltonian of one singled out (soft or critical) mode. This enables one to overcome the standard difficulties in solving the eigenvalue problem (to obtain eigen-frequencies and polarization vectors of normal lattice modes), as SPT compounds, as a rule, have complex structures. Namely, in dealing with the singled out soft mode, the dimension of the eigenvector space can be reduced significantly if one applies symmetry arguments. If the wave vector star at which a structural phase transition takes place (the critical wave vector) is known, then it is possible to determine the relevant irreducible representation for the phase transition. This approach was applied, for instance, in calculating the lattice vibration frequencies of ferroelectric $\mathrm{KH}_{2} \mathrm{PO}_{4}$ for critical wave vector $\mathbf{q}_{c}=0[9]$. As the result of a symmetry analysis, 48-dimensional eigenvector space is reduced to 7-dimensional space, in which case, one of the seven phonon modes is acoustic, while the rest of them are optical. The frequencies and displacement vectors of the six low-lying optical modes, as calculated in [9] (using a rather rough, i.e. quasiharmonic approximation), are in satisfactory agreement with the results obtained in experiments on light scattering. In this case the corresponding singled out (critical) LNC practically coincides with the displacements of hydrogen atoms in the cell, and one may consider only the motion of the so-called, "active" atoms responsible for the phase transition. It should be noted that the physical situation in $A B X_{3}$ perovskites resembles that in hydrogen-bonded ferroelectrics. Namely, the principal simplification adopted in the model description (by singling out the $R_{25}$ or $M_{3}$ mode) consists in neglecting displacements of all the ions with the exception of $X$ cations and taking account only of their displacements which lie in the face plane of the unit cell (cf. [7]).

The next step towards the model description consists in introducing the normal coordinate corresponding to the singled out soft mode (index $s$ ) of the spectrum which turns out critical at the SPT-temperature $\left(T=T_{c}\right)$ :

$$
Q_{\mathbf{q} s}=\frac{1}{\sqrt{N}} \sum_{n d \alpha} e_{\mathbf{q} s}^{\alpha}(d) \sqrt{m_{d}} u_{n d}^{\alpha} e^{-i \mathbf{q} \mathbf{x}_{n}} .
$$

The above notation is obvious from the coordinates of atoms defined herein in a more complete form (for a crystal considered in the adiabatic approximation):

$$
\mathbf{R}_{n d}=\mathbf{x}_{n d}+\mathbf{u}_{n d}=\mathbf{x}_{n d}^{0}+\boldsymbol{\eta}_{n d}+\mathbf{u}_{n d}
$$

where $\mathbf{x}_{n d}^{0}=\mathbf{n}+d$ represents the equilibrium position of the $d$-th atom in the $n$-th primitive cell in the symmetric phase; $\boldsymbol{\eta}_{n d}$ describes the change in the equilibrium positions of atoms when the crystal undergoes a transition to the nonsymmetric phase; $\mathbf{u}_{n d}$ (or taken as $\mathbf{S}_{n d}$ in Section 3) are dynamic (time dependent) displacements of atoms with respect to the equilibrium positions $\mathbf{x}_{n d}=\mathbf{x}_{n d}^{0}+\boldsymbol{\eta}_{n d} ; m_{n d}$ is the mass of the $n d$-th atom and $e_{\mathbf{q} \lambda}^{\alpha}(d)$ are polarization vectors forming an orthogonalized basis (labelled by index $\lambda$ ) in the $3 r N$-vector space, $r$ - the number 
of atoms in each primitive cell, $N$ is the total number of cells in the crystal of volume $V, \alpha=x, y, z$. Henceforth, for definiteness we shall consider transitions at the centre of the Brillouin zone $\left(\mathbf{q}_{c}=0\right)$ and introduce LNCs for the critical (soft) mode [10],

$$
x_{n s}=\sum_{d \alpha} \sqrt{m_{d}} e_{\mathbf{q}=0, s}^{\alpha}(d) u_{n d}^{\alpha} .
$$

These coordinates are local, meaning that summation in (2.3) runs over all the atoms in the $n$-th primitive cell and that it characterizes the cell as a whole. As it has been already remarked, coordinates $x_{n s}$ obey the symmetry transformations of the point group (while the order parameter - obeys those of the space group), thus, forming the basis for describing SPT. The application of coordinates $x_{n s}(2.3)$ and $Q_{\mathbf{q} s}\left(\mathbf{q} \simeq \mathbf{q}_{c}=0\right)(2.1)$ in the Brillouin zone is restricted by the range of a weak $\mathbf{q}^{-}$ dependence of the polarization vectors. As usual, in the case of optical modes the polarization vectors do not vary too much with a change in $\mathbf{q}$. The corresponding limiting value of the wave vector intensity $q_{m}$ depends on the dispersion of the Fourier-transformed force constant (in the dynamical matrix), and, consequently, on the effective interaction radius in the system $R\left(q_{m} \sim 1 / R\right)$ [10].

\subsubsection{Conceiving the model Hamiltonian}

In describing SPT systems we start with quite a general Hamiltonian:

$$
H=\sum_{i}\left[\frac{\mathbf{P}_{i}^{2}}{2 m_{i}}+U\left(\mathbf{R}_{i}\right)\right]+\frac{1}{2} \sum_{i \neq j} V\left(\mathbf{R}_{i}, \mathbf{R}_{j}\right) .
$$

Here $\mathbf{P}_{i}$ is a canonical conjugate momentum to coordinate $\mathbf{R}_{i}$ referring to every active in SPT atom $i \equiv(n, d)$ of the mas $m_{i} ; U\left(\mathbf{R}_{i}\right)$ is a single-site potential and the pair interaction potentials $V\left(\mathbf{R}_{i}, \mathbf{R}_{j}\right)$ define the critical dynamics of the system.

Since the atomic displacements with respect to the centre of the cell (henceforth designated by vector $\mathbf{l}_{i}$ ) are usually small, the single-site potential $U\left(\mathbf{R}_{i}\right)$ and the pair-potential $V\left(\mathbf{R}_{i}, \mathbf{R}_{j}\right)$ can be expanded in terms of displacements $\mathbf{u}_{i}^{\alpha}$ as follows:

$$
\begin{gathered}
V\left(\mathbf{R}_{i}^{\alpha}\right)=\sum_{n}^{\infty} \frac{1}{n !}\left(\mathbf{u}_{i}^{\alpha} \frac{\partial}{\partial \mathbf{l}_{i}}\right)^{n} U\left(\mathbf{l}_{i}\right), \\
V\left(\mathbf{R}_{i}^{\alpha}-\mathbf{R}_{j}^{\beta}\right)=\sum_{n}^{\infty} \frac{1}{n !}\left[\left(\mathbf{u}_{i}^{\alpha}-\mathbf{u}_{j}^{\beta}\right) \frac{\partial}{\partial \mathbf{l}_{i}}\right]^{n} V\left(\mathbf{l}_{i}-\mathbf{l}_{j}\right) .
\end{gathered}
$$

To the lowest approximation it suffices to keep only the first few terms in expansions (2.5) and (2.6), thus, writing a single-particle potential in the form

$$
U\left(\mathbf{R}_{i}^{\alpha}\right)=U\left(\mathbf{l}_{i}^{\alpha}\right)+\frac{1}{2} A_{i}^{\alpha}\left(\mathbf{u}_{i}^{\alpha}\right)^{2}+\frac{1}{4} B_{i}^{\alpha}\left(\mathbf{u}_{i}^{\alpha}\right)^{4}
$$

while in the pair interaction it is sufficient to retain only the harmonic term 


$$
V\left(\mathbf{R}_{i}^{\alpha}-\mathbf{R}_{j}^{\beta}\right)=V\left(\mathbf{l}_{i}^{\alpha}-\mathbf{l}_{j}^{\alpha}\right)+\frac{1}{2} \varphi_{i j}^{\alpha \beta}\left(\mathbf{u}_{i}^{\alpha}-\mathbf{u}_{j}^{\beta}\right)^{2} .
$$

In the above expressions the linear and the third order terms disappear from the equilibrium conditions (by taking $U^{\prime}\left(\mathbf{l}_{i}\right)=U^{\prime \prime \prime}\left(\mathbf{l}_{i}\right)=V^{\prime}\left(\mathbf{l}_{i}-\mathbf{l}_{j}\right)=0$ ) and the following abbreviations are used:

$$
A_{i}^{\alpha}=V^{\prime \prime}\left(\mathbf{l}_{i}^{\alpha}\right), B_{i}^{\alpha}=\frac{1}{6} V^{\prime v}\left(\mathbf{l}_{i}^{\alpha}\right) ; \quad \varphi_{i j}^{\alpha \beta}=V^{\prime \prime}\left(\mathbf{l}_{i}^{\alpha}-\mathbf{l}_{j}^{\beta}\right) .
$$

The single particle potential (2.7) may be thought of as arising from an underlying sublattice of atoms which do not participate actively in SPT. Moreover, we will assume that atomic displacements are found along a given crystal axis, although the full spectrum of lattice vibrations is referred to a three-dimensional case.

From the condition (indisputable in itself) that the minimum of the total energy in the system at zero temperature must be attained for the square value of the order parameter $\left\langle\left. u_{i d}\right|_{T=0} ^{2}=-A / B\right.$, there follows that it must be $A<0$ for positive B. Thus, a necessary condition for the existence of SPT is apparently equivalent to the demand that the single-particle potential (2.7) should be double-welled. In effect, the single-particle potential, strongly anharmonic in general, acquires the form of a quartic (double-well) oscillator having two stable atomic configurations, one of which prevails in the system at $T<T_{c}$, and yielding a homogeneously ordered phase with the order parameter $\left\langle u_{i d}\right\rangle_{T=0}= \pm(|A| / B)^{1 / 2}$ at zero temperature.

With all the aforegoing remarks, restricting ourselves to the singled out mode $(\lambda \equiv s)$, namely, using the LNC-representation (2.3), we arrive at the effective model Hamiltonian in the form:

$$
H_{s}=\sum_{i}\left[\frac{p_{i}^{2}}{2 m}-\frac{A_{i}}{2} x_{i}^{2}+\frac{B_{i}}{4} x_{i}^{4}\right]+\frac{1}{4} \sum_{i \neq i^{\prime}} \varphi_{i i^{\prime}}\left(x_{i}-x_{i^{\prime}}\right)^{2},
$$

where, for convenience, we drop subscript $s$ of the variable $x_{i s}(2.3)$. In the above Hamiltonian (2.10) $p_{i}$ is a conjugated momentum to $x_{i}, m \equiv m_{s}$ being the effective mass corresponding to the critical mode; both the harmonic and quartic constants associated with a single-site double-well potential are supposed to be site-independent, i.e. $A_{i} \equiv A$ and $B_{i} \equiv B ; \varphi_{i i^{\prime}}$ describes the coupling between the displacements of a local normal mode in cells $i$ and $i^{\prime}$ and determines the dispersion of the mode, i.e. the $q$-dependence of its frequency $\omega_{q s} \equiv \omega_{q}$. The space dimension of the model depends on the type of a lattice chosen, on which the pair-interaction $\varphi_{i i^{\prime}}$ is given. In the general case the local normal coordinate $x_{i s} \equiv x_{i}$, in accordance with definition (2.3), represents a multicomponent vector the dimension of which is determined by the dimension of the relevant irreducible representation (the number of components of polarization vector $\mathbf{e}_{\mathbf{q}=\mathbf{q}_{c}, s}$ ). For a one-component (scalar) coordinate $x_{i}$ the Hamiltonian (2.10) describes SPT in a uniaxial system*.

\footnotetext{
${ }^{*}$ Henceforth, the vector notation for direct and inverse one-dimensional $(d=1)$ spaces is omitted.
} 
In addition, it should be remarked that the one-site (cell) potential in equation (2.10) ensures a simple stabilizing interaction, since in the harmonic (index $h$ ) case $\left(B_{i} \equiv 0\right)$ the local normal mode is unstable for $A>0: \omega_{h, q=0}^{2}=-A / m$ (cf. [9]). To avoid any confusion, it should be noted that the dimensionalities of parameters A,B and $\varphi_{i i^{\prime}}$ in equation (2.10) are modified in accord with the dimensions of variables $x_{i}$ and $p_{i}$, which is otherwise immaterial for "the essentials" of the unified model description of SPT.

Thus, the Hamiltonian (2.10) represents a dynamic microscopic model of SPT in the reduced space of coordinates related directly to the phase transition. It is the Hamiltonian of one singled out mode, namely, of the critical mode. As we have already mentioned, its interaction with the remaining modes, if allowed by the system symmetry, can also be taken into account in terms of local normal coordinates.

\subsubsection{The soft-acoustic mode interaction}

At this stage it should be stressed once more that at many SPTs the size and shape of the unit cell alter with temperature. These changes are characterized by the infinitesimal strain tensor $u_{\alpha \beta}$, provided that the atomic displacements brought about by the lattice deformation are represented as

$$
\eta_{n d}^{\alpha}=\sum_{\beta} u_{\alpha \beta} x_{n d}^{\beta}
$$

Using a general expression for the normal coordinate $Q_{q \lambda}$ such as (2.1) (with running index $\lambda \leqslant 3 r N$ instead of the singled out one $(s)$ indicating the soft mode) together with (2.11), one obtains

$$
u_{\alpha \beta}=\lim _{q_{\beta} \rightarrow 0} \frac{i q_{\beta}}{\sqrt{N \sum_{d} m_{d}}} Q_{q_{\beta} \alpha},
$$

where $Q_{q_{\beta} \alpha}$ is an amplitude of the acoustic mode with its eigenvector polarized in the $\alpha$-direction and its wave-vector projected onto the $\beta$-direction. However, no particular combination of strain parameters can be expressed in terms of the amplitudes of the acoustic mode. Consequently, the terms containing a long-wavelength acoustic mode (namely, the strains) must be eliminated from the summation over indices $\mathbf{q} \lambda$ in the initial harmonic Hamiltonian (in terms of normal coordinates and momenta) and written as an additional term to the Hamiltonian (2.10) in the form showing the relationship between the critical mode (i.e. the LNC) and the lattice deformation (index $D)$ (cf. $[7,11])$ :

$$
H_{D}=\sum_{i, \alpha \beta} u_{\alpha \beta}\left[U_{1 s}^{\alpha \beta} x_{i s}+U_{2 s}^{\alpha \beta} x_{i s}{ }^{2}\right]+\frac{V_{o}}{2} \sum_{\alpha \beta \gamma \delta} u_{\alpha \beta} u_{\gamma \delta}\left[C_{\alpha \beta \gamma \delta}+\ldots\right] .
$$

In the above expression (2.13) only three most important terms are written out of the double power series expansion around the equilibrium positions of the sym- 
metric phase. The first term in equation (2.13) describes the coupling of the internal homogeneous deformation (the optic mode of vibrations in the long-wavelength limit) to the external homogeneous deformation determined by the last term which contains the product of the usual elastic constants $C_{\alpha \beta \gamma \delta}$ and the strain variables multiplied by the volume of the unit cell $V_{o}=V / N$.

In what follows we neglect variations both in the shape and size of the unit cell with temperature and pressure, i.e. we use the standard approximation of a "clamped" crystal. As it was pointed out in [11], even with this oversimplification including less drastic ones (such as the uniaxial anisotropy, the absence of the thirdorder "site"-anharmonicity, short-range forces, etc.), most of the basic features of the theory of SPT are still comprised in the simple model Hamiltonian (2.10) that does offer a unified approach in describing the order-disorder and displacive systems - a description which is to be regarded as an inherent expression of their universal (Ising-like) critical behaviour.

\subsubsection{The essential features of the model}

The microscopic model Hamiltonian (2.10) is characterized by two energy parameters: the depth of the potential well

$$
U_{o}=A^{2} / 4 B,
$$

and the relative binding energy of the particles

$$
U_{c}=\frac{\varphi_{o}|A|}{B}, \quad \varphi_{o}=\sum_{n^{\prime}} \varphi_{n n^{\prime}}
$$

as well as by a parameter describing the quantum properties of the system,

$$
\lambda=\hbar \omega_{o} / 4 U_{o}, \quad \omega_{0}=\sqrt{A / m} .
$$

The parameter $\lambda \sim \hbar / \sqrt{m}$ determines a relative zero-point energy of vibrations. If the system is characterized by a large zero-point energy of vibrations, so that $\lambda$ is greater than a certain critical value $\lambda_{c}$, then the effective one-particle potential in (2.10) turns into a harmonic potential with a single minimum (see subsection 4.54.7). In this case no SPT occurs even at zero temperature (in strontium titanate, for example).

When $\lambda \leqslant \lambda_{c}$, the Hamiltonian (2.10) describes two extreme cases of SPT: displacive and order-disorder, depending on the parameter

$$
f_{0} \equiv U_{c} / 4 U_{o}=\varphi_{0} /|A| .
$$

The structural transformations are called displacive transitions under which in the non-symmetric phase atoms are displaced insignificantly (by several percent of the lattice constant) with respect to their equilibrium positions in the symmetric phase (for example, at the ferroelectric transition in $\mathrm{BaTiO}_{3}$ ). The notion of a soft phonon mode was originally attributed to displacive transitions, which 
is based on extensive experimental observations (cf. in [7]). Displacive transitions are described by model $(2.10)$ for $f_{0} \gg 1$ or $T_{c} \ll U_{0}$. It is usual to consider the displacive transitions as being characterized by resonance dynamics, although in this case the critical mode may even undergo strong damping. The latest studies of the nonlinear properties of the model have revealed that in the critical region a displacive transition acquires the features of an order-disorder transition $\left(f_{0} \ll 1\right.$ or $T_{c} \ll U_{0}$ ) (see section 5 ).

Order-disorder transitions are such transitions at which there arises a longdistance order owing to the ordering of certain atomic complexes in the nonsymmetric phase (for example, the orientational ordering of $\mathrm{NO}_{2}$ groups at a ferroelectric transition in $\mathrm{NaNO}_{2}$ ). Reorientations of $\mathrm{NO}_{2}$ groups involve quite large displacements of atoms ( $N$ ions) relative to the equilibrium positions, so no expansion in these displacements can be performed. In such cases the notion of a soft mode does not represent the physical situation adequately, since the dynamics in the critical region is of a relaxational character. This is almost the situation envisaged in the Ising model of ferromagnetism. Otherwise, to an order-disorder transition there corresponds a phase transition in the Ising model which is obtained from model (2.10) when $f_{0} \rightarrow 0(A, B \rightarrow \infty$ for $A / B=$ const) [12]. Besides, it is this equivalency of the two models (model $(2.10)$ - often called " $\phi{ }^{4}$-model" and the Ising model with a transverse field - abbreviated as IMTF hereafter) which motivated the introduction of pseudospin formalism (i.e. various versions of the adopted pseudospin - phonon Hamiltonians) into the underlying unified SPT description.

The division of SPTs into two types is based on quite sound thermodynamical arguments (cf. $[7,11]$ and Refs. therein). However, in considering lattice dynamics such a division is only conventional. There exists a broad class of systems, for example, ferroelectrics of the $\mathrm{KH}_{2} \mathrm{PO}_{4}$ type, which are difficult to attribute to any of the limiting types, since from spectral measurements it is not clear whether the critical mode exhibits the character of an overdamped soft mode or of a relaxational mode.

\section{The unified description of strongly anharmonic systems}

\subsection{Preamble: convenience of simple approximations}

As we have already mentioned, it is generally assumed that there are two basic kinds of SPTs (ferroelectric included), one being of the order-disorder type and the other being of the displacive type. In the former case the SPT results from a statistical or tunnelling induced disorder of atoms among several (in the simplest case, two) equilibrium positions. In the latter case SPT is caused by lattice instability against a critical vibrational mode (soft mode). Nevertheless, it was shown about a quarter century ago that the both types of SPT can be described within a single model (2.10) and there are no essential differences between them (cf. [1]). The nature of the SPT described within such a model was examined by applying 
both the Curie-Weiss (molecular-field) and the self-consistent phonon approximations ([cf. $[1,2,7])$. By comparing the results of the both approximations it has been shown that for a weak lattice coupling $\left(f_{0} \ll 1\right)$ the character of the SPT is of the order-disorder type, which is more consistently described by the molecular-field approximation; for a strong lattice coupling $\left(f_{0} \gg 1\right)$ the SPT has to be related to the displacive type, which can be reasonably described by the self-consistent phonon approximation. Such a consistent description can be understood under the circumstances that in the order-disorder transition statistical or tunnelling fluctuations of atoms onto their equivalent equilibrium position play the main role, which is accurately enough described by a pseudospin model; while in the displacive transition the dynamical correlations of atomic displacements turn out to be more essential, so the self-consistent phonon approximation is more adequate. The structural transition itself can also have a mixed character, depending on the relations between the energy parameters introduced in the model.

For a complete description of SPT one has to take into account the both above mentioned mechanisms simultaneously - within the framework of a universal model - as was originally proposed and done in $[1,2]$. In the next section we are going to present the main features and results of such a description.

\subsection{The adopted model Hamiltonian and equilibrium conditions}

In the case of a relatively deep double-well local potential $U\left(x_{i}\right)(2.7)$ with a negligible tunnelling of atoms between the two minima, atomic (i.e. cell) oscillations inside the wells are of finite frequency which cannot be ignored. Therefore, apart from two degenerate eigenstates per cell (one near the bottom of each sitepotential) entering the statistical problem, one has to take into account these intra-well oscillations, too, so that the inter-well tunnelling is entirely neglected. Besides, the two equilibrium positions of active atoms (namely, in each cell) are randomly distributed in the lattice and apparently determined by a self-consistent account of the vibrational and configurational parts of free energy. For this reason it is convenient to represent the effective time-dependent displacement $x_{i} \equiv x_{i s}$ (2.3) as follows (compare with equation (2.2)):

$$
x_{i}(t)=r_{i}(t)+u_{i}(t) .
$$

As dynamic displacements, $r_{i}$ have their particular value for each cell $(i)$ and are practically quasiequilibriums, meaning that they can change with time. The dynamical displacement $u_{i}(t)$ is associated with the atomic vibrations around momentary rest positions $r_{i}(t)$. Below $T_{c}$ there are two "stable" configurations $r_{\alpha}=\alpha\left\langle r_{i}\right\rangle(\alpha=-,+)$, and above $T_{c}$ the system is to be found in one as well as the other. However, in the case of strong anharmonic systems more appropriate is to introduce, right from the beginning, the projection operator $\sigma_{i}^{\alpha}$ onto two states in the "left" hand (-) or the "right" hand (+) parts of one-site potential (2.7) and thus to represent the LNC (3.1) in the form: *

\footnotetext{
*One has to distinguish between state index $(\alpha=-,+)$ and coordinate index $(\alpha=x, y, z)$.
} 


$$
x_{i}=\sum_{\alpha= \pm 1} x_{i}^{\alpha} \sigma_{i}^{\alpha} .
$$

For convenience we also introduce variables $S_{i}$ and $b_{i}$ as alternatives to variables $x_{i}$ and $r_{i}$ (in equation (3.1)), respectively. Then, representation (3.2) in new variables $S_{i}$ reads:

$$
S_{i}=\sum_{\alpha= \pm} S_{i}^{\alpha} \sigma_{i}^{\alpha}
$$

The projection operators $\sigma_{i}^{\alpha}$ themselves are expressed, as usual, through the operators of pseudospin (the Pauli operator) $\sigma_{i}^{z}$, being independent variables which commute with coordinates $x_{i}^{\alpha}$ and the corresponding momenta $p_{i}^{\alpha}$ :

$$
\sigma_{i}^{\alpha}=\frac{1}{2}\left(1+\alpha \sigma_{i}^{z}\right)
$$

The coordinate in state $\alpha, S_{i}^{\alpha}$, can be written as a sum of static displacement $b_{i}^{\alpha}$ and thermal fluctuation $u_{i}^{\alpha}$ :

$$
\begin{array}{r}
S_{i}^{\alpha}=b_{i}^{\alpha}+u_{i}^{\alpha} \\
b_{i}^{\alpha}=\left\langle S_{i}^{\alpha}\right\rangle=b_{\alpha},
\end{array}
$$

where symbol $\langle\ldots\rangle$ stands for a statistical average with the Hamiltonian (2.10). The representation of atomic distortions (3.3)-(3.5) enables one to take into account, firstly, the atomic random distribution over two equilibrium positions in the cell, using operator $\sigma_{i}^{\alpha}$, and, secondly, the thermal fluctuation $u_{i}^{\alpha}$ in the neighbourhood of a given equilibrium position. In describing order-disorder SPT the variables $u_{i}^{\alpha}$ are usually neglected, whereas in displacive SPT only one equilibrium position in the cells $(\alpha=+1$ or $\alpha=-1)$ is assumed, meaning that operator $\sigma_{i}^{\alpha}$ takes the same value at each lattice site $i$.

In this generalized model we will be able to study the both types of SPT using full representation (3.3)-(3.5). It should be noted that a similar representation for atomic coordinates was proposed by Vaks and Larkin [12] in discussing the orderdisorder type SPT. We generalize their representation to consider the displacive type SPT as well.

Having inserted expression (3.2), i.e. (3.3), into the model Hamiltonian (2.10), it can be written in the form:

$$
H=\sum_{i \alpha= \pm 1} \sigma_{i}^{\alpha}\left[\frac{1}{2 m}\left(P_{i}^{\alpha}\right)^{2}-\frac{A}{2}\left(S_{i}^{\alpha}\right)^{2}+\frac{B}{4}\left(S_{i}^{\alpha}\right)^{4}\right]+\frac{1}{2} \sum_{i, j} \sum_{\alpha, \beta= \pm 1} \sigma_{i}^{\alpha} \sigma_{j}^{\beta} \varphi_{i j} \frac{\left(S_{i}^{\alpha}-S_{j}^{\beta}\right)^{2}}{2}
$$

where $\varphi_{i j}^{\alpha \beta}(2.9)$ is assumed to be independent of $\alpha$ and $\beta$, i.e. $\varphi_{i j}^{\alpha \beta} \equiv \varphi_{i j}$.

The equilibrium positions of lattice atoms $b_{\alpha}=\left\langle S_{i}^{\alpha}\right\rangle$ are determined from the equilibrium conditions 


$$
i(\partial / \partial t)\left\langle P_{i}^{\alpha}(t)\right\rangle=\left\langle\left[P_{i}^{\alpha}, H\right]\right\rangle=0,
$$

which leads to the equation

$$
\left\langle\frac{\partial}{\partial S_{i}^{\alpha}} U\left(S_{i}^{\alpha}\right)\right\rangle+\sum_{j \beta}\left\langle\sigma_{j}^{\beta} \frac{\partial}{\partial S_{i}^{\alpha}} \varphi_{i j} \frac{\left(S_{i}^{\alpha}-S_{j}^{\beta}\right)^{2}}{2}\right\rangle=0 .
$$

By using the molecular-field approximation for a pseudospin subsystem and assuming its independence of the phonon subsystem,

$$
\sum_{\beta}\left\langle\sigma_{j}^{\beta}\left(S_{i}^{\alpha}-S_{j}^{\beta}\right)\right\rangle=\left\langle S_{i}^{\alpha}\right\rangle-\sum_{\beta} \sigma_{\beta}\left\langle S_{j}^{\beta}\right\rangle=\left(b_{\alpha}-b_{-\alpha}\right) \sigma_{-\alpha}
$$

the equilibrium conditions (3.8) may be rewritten in the form:

$$
A b_{\alpha}+B\left\langle\left(S_{i}^{\alpha}\right)^{3}\right\rangle+\left(b_{\alpha}-b_{-\alpha}\right) \sigma_{-\alpha} \sum_{j} \varphi_{i j}=0 .
$$

Having chosen the positive direction of the displacements along the mean value $b_{+}$and using the approximation

$$
\left\langle\left(S_{i}^{\alpha}\right)^{3}\right\rangle \approx b_{\alpha}^{3}+3 b_{\alpha}\left\langle\left(u_{i}^{\alpha}\right)^{2}\right\rangle
$$

the equilibrium conditions (3.10) become

$$
\eta_{\alpha}^{3}-\left(1-3 y_{\alpha}\right) \eta_{\alpha}+\left(\eta_{+}+\eta_{-}\right) f_{0} \sigma_{-\alpha}=0
$$

Here dimensionless quantities are introduced:

$$
\begin{array}{r}
\eta_{\alpha}^{2}=(B / A) b_{\alpha}^{2}, y_{\alpha}=(B / A)\left\langle\left(u_{i}^{\alpha}\right)^{2}\right\rangle, \\
f_{q}=\frac{1}{A} \sum_{j} \varphi_{j} e^{i q\left(l_{i}-l_{j}\right)}, \\
f_{0}=f_{q=0},
\end{array}
$$

and the average population of state $\alpha$ :

$$
\sigma_{\alpha}=\left\langle\sigma_{i}^{\alpha}\right\rangle=\frac{1}{2}\left(1+\alpha \sigma^{z}\right)
$$

in agreement with expression (3.4)

The analysis of the equilibrium conditions (3.12) shows that in addition to the zero solution $\eta_{+}=\eta_{-}=0$, corresponding to the symmetric phase, the solutions $\eta_{\alpha} \neq 0$ are also possible. In the case of small values, $f_{0} \ll 1$, the two equilibrium positions can exist, the magnitudes of which are close to one another $\left(\eta_{+}-\eta_{-} \simeq\right.$ $\sigma f_{0} \ll 1$ ), and there is also the solution $\sigma=0$, corresponding to a complete disorder for an order-disorder phase transition. For values of the reduced coupling parameter $f_{0} \gtrsim 0.25$, only one nonzero solution can exist at all temperatures, for example, $\eta_{+} \neq 0$ (for a complete atomic order, $\sigma=+1$ ). In this region of the coupling parameter only a displacive phase transition is possible. 


\subsection{The phonon subsystem}

The phonon spectrum and the average values of the atomic (cell) displacement correlation functions can be determined using the Green function method developed in the theory of strongly anharmonic crystals (cf. $[1,8,13]$ and Refs. therein). Consider a displacement operator Green function of the general type:

$$
D_{i j}\left(t-t^{\prime}\right)=\left\langle\left\langle u_{i}(t) ; u_{j}\left(t^{\prime}\right)\right\rangle\right\rangle=\int_{-\infty}^{\infty} \frac{\mathrm{d} \omega}{2 \pi} e^{-i \omega\left(t-t^{\prime}\right)} D_{i j}(\omega),
$$

where ordinary notation is used. The above Green function describes the atomic displacement correlations at the lattice sites $i$ and $j$, in arbitrary states, because here $u_{i}=\sigma_{i}^{+} u_{i}^{+}+\sigma_{i}^{-} u_{i}^{-}$. Let us also introduce a Green function for the fixed atomic state $\alpha$ at the site $i$ by inserting $\sigma_{i}^{\alpha}=1$ :

$$
D_{i(\alpha), j}\left(t-t^{\prime}\right)=\left\langle\left\langle u_{i}^{\alpha}(t) ; u_{j}\left(t^{\prime}\right)\right\rangle\right\rangle,
$$

which is necessary for the definition of the average quadratic atomic displacement in state $\alpha$,

$$
\left\langle\left(u_{i}^{\alpha}\right)^{2}\right\rangle=\int_{0}^{\infty} \mathrm{d} \omega \operatorname{coth} \frac{\omega}{2 k_{\mathrm{B}} T}\left\{-\frac{1}{\pi} \operatorname{Im}_{i(\alpha), i}(\omega+\underline{i} \varepsilon)\right\} .
$$

Taking into account the fact that the Green functions (3.15) and (3.16) contain the statistical average with the full Hamiltonian (3.6), thus also including the average over all the atomic states, and since those functions depend only on the difference between the atomic coordinates $S_{i}-S_{j} \simeq l_{i}-l_{j}$, we will write their Fourier expansion in terms of the reciprocal lattice vectors $q$ as follows:

$$
D_{i(\alpha), j}(\omega)=\frac{1}{N A} \sum_{q} e^{\underline{i} q\left(l_{i}-l_{j}\right)} D_{q}^{\alpha}(\omega) .
$$

The equation of motion for the Green function (3.15), using Hamiltonian (3.6), has the form:

$$
\begin{array}{r}
-m \frac{\mathrm{d}^{2}}{\mathrm{~d} t^{2}} D_{i(\alpha), j}\left(t-t^{\prime}\right)=\delta_{i j} \delta\left(t-t^{\prime}\right)- \\
-\left(A-\sum_{k} \varphi_{i k}\right)\left\langle\left\langle S_{i}^{\alpha} ; u_{j}\left(t^{\prime}\right)\right\rangle\right\rangle+B\left\langle\left\langle\left(S_{i}^{\alpha}\right)^{3} ; u_{j}\left(t^{\prime}\right)\right\rangle\right\rangle- \\
-\sum_{k \gamma} \varphi_{i k}\left\langle\left\langle\sigma_{k}^{\gamma} S_{k}^{\gamma} ; u_{j}\left(t^{\prime}\right)\right\rangle\right\rangle .
\end{array}
$$

The Green function $\left\langle\left\langle\sigma_{k}^{\gamma} S_{k}^{\gamma} ; u_{j}\left(t^{\prime}\right)\right\rangle\right\rangle$, on the right-hand side, describes the correlation of atomic displacements at sites $k$ and $j$, under the condition that the atom at site $i$ is in state $\alpha$ (for instance $\sigma_{i}^{\alpha}=1$ ). However, since $k \neq i$, one can neglect the correlation between states $\gamma$ and $\alpha$ for the atoms at sites $k$ and $i$, thus annihilating the latter condition, i.e. it is possible to use the molecular-field 
approximation for the pseudospin subsystem. In addition, the approximation of independence between the phonon and pseudospin subsystems yields:

$$
\left.\left\langle\left\langle\sigma_{k}^{\gamma} S_{k}^{\gamma} ; u_{j}\right\rangle\right\rangle\right|_{\sigma_{i}^{\alpha}=1} \simeq\left\langle\left\langle\sigma_{k}^{\gamma} S_{k}^{\gamma} ; u_{j}\right\rangle\right\rangle \simeq<\sigma_{k}^{\gamma}>\left\langle\left\langle S_{k}^{\gamma} ; u_{j}\right\rangle\right\rangle .
$$

Now, having inserted $S_{i}^{\alpha}(3.3)$ in the Green function and using the familiar pseudoharmonic approximation,

$$
\left\langle\left\langle\left(u_{i}^{\alpha}\right)^{3} ; u_{j}\right\rangle\right\rangle \simeq 3\left\langle\left(u_{i}^{\alpha}\right)^{2}\right\rangle\left\langle\left\langle u_{i}^{\alpha} ; u_{j}\right\rangle\right\rangle,
$$

for the Fourier $(q, \omega)$ - component of the Green function in equation (3.19), one obtains:

$$
D_{q}^{\alpha}(\nu)=\frac{\nu^{2}-\nu_{-\alpha}^{2}}{\left(\nu^{2}+\nu_{q+}^{2}\right)\left(\nu^{2}-\nu_{q-}^{2}\right)-\sigma_{+} \sigma_{-} f_{q}^{2}}=\frac{\nu^{2}-\nu_{-\alpha}^{2}}{\left(\nu^{2}-\nu_{q 1}^{2}\right)\left(\nu^{2}-\nu_{q 2}^{2}\right)},
$$

where reduced frequencies are introduced:

$$
\nu^{2}=\omega^{2} /(A / M) ; \quad \nu_{\alpha}^{2}=\Delta_{\alpha}^{2}+f_{0} ; \quad \nu_{q \alpha}^{2}=\nu_{\alpha}^{2}-\sigma_{\alpha} f_{q}
$$

the gap appearing in the phonon spectrum $\Delta_{\alpha}$ is determined by a single-particle potential, so that

$$
\Delta_{\alpha}^{2}=3\left(\eta_{\alpha}^{2}+y_{\alpha}\right)-1
$$

the phonon frequencies $\nu_{q(+,-)}$ in expression (3.22) correspond to atomic vibrations in the "right-hand" $(+)$ or "left-hand" (-) equilibrium positions.

If disorder is present in the system, the phonon spectrum, as determined by the Green function poles in equation (3.22), has two branches,

$$
\nu_{q(1,2)}^{2}=\frac{1}{2}\left(\nu_{q+}^{2}+\nu_{q-}^{2}\right) \pm \frac{1}{2}\left[\left(\nu_{q+}^{2}-\nu_{q-}^{2}\right)+\left(1-\sigma^{z 2}\right) f_{q}^{2}\right]^{1 / 2} .
$$

However, in the limiting case of a complete order (for instance, $\sigma^{+}=1$ and $\left.\sigma^{-}=0\right)$, the Green function (3.22) becomes

$$
D_{q}^{+}(\nu)=\left[\nu^{2}-\left(\Delta_{+}^{2}+f_{0}-f_{q}\right)\right]^{-1},
$$

and has only one pole corresponding to the vibrations of all the atoms in the "right-hand" equilibrium positions. For $\sigma^{z}=0$ the number of atoms (cells) in both states becomes equal, $\sigma_{+}=\sigma_{-}=\frac{1}{2}$, so the average field at each site takes the same value: $\Delta_{+}^{2}=\Delta_{-}^{2}=\Delta_{0}^{2}$. Therefore, the phonon spectrum in this case is determined by a single frequency being the pole of the Green function

$$
D_{q}^{\alpha}(\nu)=\left[\nu^{2}-\left(\Delta_{0}^{2}+f_{0}-f_{q}\right)\right]^{-1} .
$$

Hence, in both cases a soft mode emerges when the single-particle gap (3.24) vanishes, $\Delta_{\alpha}^{2} \rightarrow 0$. 
A Green function of the general type (3.15) which can be obtained from equation (3.22), using approximation (3.20), evidently has the same properties.

The last self-consistent equation for the phonon subsystem represented by a phonon self-correlation function (3.17), in the high-temperature (classical) limit can readily be expressed in a simple form:

$$
\begin{aligned}
& y_{\alpha}=\frac{B}{A}\left\langle\left(u_{i}^{\alpha}\right)^{2}\right\rangle= \\
&=\frac{B}{N A^{2}} \sum_{q} \int_{0}^{\infty} \mathrm{d} \omega \operatorname{coth} \frac{\omega}{2 k_{\mathrm{B}} T}\left[-\frac{1}{\pi} \operatorname{Im} D_{q}^{\alpha}(\omega+\underline{i} \varepsilon)\right] \simeq \\
& \simeq \frac{\tau}{N} \sum_{q} \int_{-\infty}^{\infty} \frac{\mathrm{d} \nu}{\nu}\left[-\frac{1}{\pi} \operatorname{Im} D_{q}^{\alpha}(\nu+\underline{i} \varepsilon)\right]= \\
&=-\frac{\tau}{N} \sum_{q} \operatorname{Re} D_{q}^{\alpha}(0+\underline{i} \varepsilon)=\frac{\tau}{N} \sum_{q} \frac{\nu_{-\alpha}^{2}}{\nu_{q 1}^{2} \nu_{q 2}^{2}},
\end{aligned}
$$

where the reduced temperature is given by

$$
\tau=\frac{k_{\mathrm{B}} T}{\left(A^{2} / B\right)} .
$$

It is convenient to pass from the summation over $q$ in the first Brillouin zone to the integration over frequencies by introducing the familiar frequency spectrum density

$$
g\left(\omega^{2}\right)=\frac{1}{N} \sum_{q} \delta\left(f_{0}-f_{q}-\omega^{2}\right) .
$$

Taking into account expressions (3.23) and (3.25), equation (3.28) can be rewritten in the form:

$$
y_{\alpha}=\tau \int_{0}^{\infty} \frac{g\left(\omega^{2}\right) \mathrm{d} \omega^{2}}{P+Q \omega^{2}}\left[\Delta_{-\alpha}+f_{0}\right]
$$

where the following abbreviations are introduced:

$$
\begin{aligned}
P & =\Delta_{+}^{2} \Delta_{-}^{2}+f_{0}\left[\Delta_{+}^{2} \frac{1}{2}\left(1+\sigma^{z}\right)+\Delta_{-}^{2} \frac{1}{2}\left(1-\sigma^{z}\right)\right] \\
Q & =f_{0}+\Delta_{+}^{2} \frac{1}{2}\left(1-\sigma^{z}\right)+\Delta_{-}^{2} \frac{1}{2}\left(1+\sigma^{z}\right) .
\end{aligned}
$$

Finally, performing an integration in equation (3.28) in the case of zero temperature (when $\operatorname{coth}\left(\omega / 2 k_{\mathrm{B}} T\right)=1$ ) and taking into account equation (3.22), one obtains for the quantum limit $(T=0)$ :

$$
y_{\alpha}=\frac{\lambda}{N} \sum_{q} \frac{1}{2\left(\nu_{q 1}+\nu_{q 2}\right)}\left[1+\frac{\Delta_{-\alpha}^{2}+f_{0}}{\nu_{q 1} \nu_{q 2}}\right],
$$


where quantum parameter $\lambda$ is defined by equation (2.16).

Now, substituting $y_{\alpha}$ (equation (3.30)) (or - in the quantum limit - equation (3.32)) and $\Delta_{\alpha}^{2}(3.24)$ in the equilibrium condition (3.12) we arrive at a selfconsistent procedure to determine the order parameter $\eta_{ \pm}$, provided that the order parameter $\sigma^{z}$ is to be independently found from the analysis of the pseudospin subsystem.

\subsection{The pseudospin subsystem}

Besides phonon variables $\left(u_{n}\right)$, the Hamiltonian (3.6) also contains configuration variables (3.4), therefore, the equilibrium values of pseudospin $\sigma^{z}$ (3.14) can be directly calculated from the minimum of the total free energy. However, calculation of the trace over the pseudospin and phonon variables for the density matrix with the Hamiltonian (3.6) is a rather difficult task, since the phonon and pseudospin variables cannot be separated. This fact is easily verified by substituting (3.4) into (3.6). As a result, we obtain the Hamiltonian:

$$
\begin{aligned}
H & =H_{l}+H_{s}, \\
H_{s} & =\sum_{n} h_{n} \sigma_{n}^{z}-\frac{1}{2} \sum_{n \neq n^{\prime}} J_{n n^{\prime}} \sigma_{n}^{z} \sigma_{n^{\prime}}^{z},
\end{aligned}
$$

where $H_{l}$ is independent of $\sigma_{n}^{z}$, while the parameters $h_{n}$ and $J_{n n^{\prime}}$ in $H_{s}$ contain phonon variables in an explicit manner, as is entirely dictated by the Hamiltonian (3.6). We shall take advantage of the Bogolyubov variational principle for free energy, applying trial Hamiltonian for a pseudospin subsystem:

$$
\tilde{H}_{s}=\sum_{n} \tilde{h}_{n} \sigma_{n}^{z}-\frac{1}{2} \sum_{n \neq n^{\prime}} \tilde{J}_{n n^{\prime}} \sigma_{n}^{z} \sigma_{n^{\prime}}^{z},
$$

where the effective mean field $\tilde{h}_{n}$ and the exchange energy $\tilde{J}_{n n}$, no longer depend explicitly on the phonon variables. Parameters $\tilde{h}_{n}$ and $\tilde{J}_{n n^{\prime}}$ are determined from the variational equations $\delta F_{1} / \delta \tilde{J}_{n n^{\prime}}=0$ and $\delta F_{1} / \delta \tilde{h}_{n}=0$, where $F_{1}=F_{0}+$ $\left\langle H-H_{0}\right\rangle_{H_{0}}$ is the trial free energy with the Hamiltonian $H_{0}=H_{l}+\tilde{H}_{s}$, and is of the form $[7,14]$ :

$$
\begin{gathered}
\tilde{h}_{n}=\sum_{\alpha} \alpha\left[\frac{\left\langle\left(p_{n}^{\alpha}\right)^{2}\right\rangle}{4 m}+\frac{A}{4} \Delta_{\alpha}\left\langle\left(u_{n}^{\alpha}\right)^{2}\right\rangle\right]+\frac{1}{8} \sum_{\alpha \beta n^{\prime}} \alpha \varphi_{n n^{\prime}}\left\langle\left(u_{n}^{\alpha}-u_{n^{\prime}}^{\beta}\right)^{2}\right\rangle ; \\
\tilde{J}_{n n^{\prime}}=\frac{A}{4 B} \varphi_{n n^{\prime}}\left(\eta_{+}+\eta_{-}\right)^{2}-\frac{1}{8} \sum_{\alpha \beta} \alpha \beta \varphi_{n n^{\prime}}\left\langle\left(u_{n}^{\alpha}-u_{n^{\prime}}^{\beta}\right)^{2}\right\rangle .
\end{gathered}
$$

The effective mean field $\tilde{h}_{n}$ depends on the vibrational energy difference between the states $(+)$ and $(-)$. The effective exchange energy depends on the distance $\left(\eta_{+}+\eta_{-}\right)$between the equilibrium positions of the particles in a cell and also on the phonon correlation function. 
In the case of high temperatures, taking into account the solution of the Green function (3.22) and the approximative equality $\left\langle P_{\alpha}^{2}\right\rangle \sim\left(k_{B} T\right) m$, the effective field $\tilde{h}_{n}$ and the effective exchange energy, become, respectively:

$$
\begin{gathered}
\tilde{h}_{n}=\frac{A^{2}}{4 B}\left\{\frac{1}{6}\left(\Delta_{+}^{4}-\Delta_{-}^{4}\right)-\left(\eta_{+}^{4}-\eta_{-}^{4}\right)-\frac{1}{Q}\left[P\left(y_{+}-y_{-}\right)+\tau\left(\Delta_{+}^{2}-\Delta_{-}^{2}\right)\right]\right\}, \\
\tilde{J}_{n n^{\prime}}=\frac{A}{4 B} \varphi_{n n^{\prime}}\left(\eta_{+}+\eta_{-}\right)^{2} .
\end{gathered}
$$

As it is easily seen, $\tilde{h}$ plays the role of a mean field caused by thermal atomic vibrations (when $\tau \rightarrow 0, \tilde{h} \rightarrow 0$ ) which tends to zero if $\sigma^{z} \rightarrow 0$. The effective exchange energy (3.38) defined by the equilibrium atomic positions turns into zero above the critical (SPT) temperature, when $\eta_{ \pm}=0$, leading to the unique solution $\sigma^{z} \equiv 0$.

By using the molecular-field approximation, for the order parameter $\sigma^{z}$ one obtains:

$$
\sigma^{z}=\tanh \left(\frac{\tilde{J}_{0} \sigma^{z}-\tilde{h}}{k_{\mathrm{B}} T}\right) ; \quad \tilde{J}_{0}=\sum_{j} \tilde{J}_{i j}
$$

or having taking into account definitions (3.37) and (3.38) the above equation (3.39) acquires the explicit form:

$$
\begin{array}{r}
\sigma^{z}=\tanh \left\{\frac { 1 } { 4 \tau } \left(\sigma ^ { z } f _ { 0 } \left(\eta_{+}+\right.\right.\right. \\
\left.+\eta_{-}\right)^{2}+\left(\eta_{+}^{4}+\eta_{-}^{4}\right)-\frac{1}{6}\left(\Delta_{+}^{4}-\Delta_{-}^{4}\right)+ \\
\left.\left.+\frac{1}{Q}\left[P\left(y_{+}-y_{-}\right)+\tau\left(\Delta_{+}^{2}-\Delta_{-}^{2}\right)\right]\right)\right\} .
\end{array}
$$

Thus, the system of self-consistent equations for parameters $\eta_{ \pm}(3.12)$ and $\sigma^{z}$ (3.40) becomes complete, where quantities $y_{ \pm}$and $\Delta_{ \pm}^{2}$ are defined by the suitable functions (3.30) and (3.24), respectively.

In addition, the expression for the spontaneous polarization per atom (i.e. per cell) - the total order parameter of the system should be quoted. It is determined by both subsystem-order parameters, $\eta_{ \pm}$and $\sigma^{z}$. In dimensionless quantities the spontaneous polarization is given by

$$
\begin{array}{r}
P_{s}=\frac{1}{N} \sum_{n}\left(\frac{B}{A}\right)^{1 / 2}\left(\left\langle\sigma_{n}^{+} S_{n}^{+}\right\rangle-\left\langle\sigma_{n}^{-} S_{n}^{-}\right\rangle\right)= \\
=\frac{1}{2}\left(\eta_{+}-\eta_{-}\right)+\frac{1}{2} \sigma^{z}\left(\eta_{+}+\eta_{-}\right) .
\end{array}
$$

If $\tau=0$, then it follows that $\sigma^{z}=1$, so the polarization takes its maximum value $P_{s}=1$; but if $\sigma^{z} \rightarrow 0$, then it is clear that $P_{s} \rightarrow 0$. 


\subsection{The limiting types of SPT}

In a general case the system of self-consistent equations obtained for the order parameters $\eta_{ \pm}$and $\sigma^{z}$ can only be solved numerically. Nevertheless, even a quantitative analysis of equations - in limiting cases - enables one to draw some definite general conclusions.

At sufficiently low temperatures, $\tau \ll \tau_{s}$ ( $\tau_{s}$ is a dimensionless lattice-instability temperature), it is possible to neglect the influence of lattice vibrations on the pseudospin subsystem and to consider only equation (3.40), with $\eta_{+} \simeq \eta_{-}=1$. In this case we have a well-known Ising model in which the phase transition of the order-disorder type (second order) takes place at temperature $\tau_{k}=f_{0}$ (using the molecular-field approximation, equation (3.40)). As we will see later, this result holds only if $f_{0} \ll 1$.

By neglecting the temperature dependence of parameter $\sigma^{z}(\tau)$, hereafter, we consider the limiting cases $\sigma^{z}=1$ and $\sigma^{z}=0$ (see subsection 3.6).

If $\sigma^{z}=1$, for quantity $\Delta_{+}^{2} \equiv \Delta^{2}(\tau)$, from equations (3.12), (3.24) and (3.30) (in the nonsymmetric phase $\eta_{+} \equiv \eta \neq 0$ ) one obtains the following equation:

$$
\Delta^{2}=2 \eta^{2}=2-6 \tau \int_{0}^{\infty} \frac{g\left(\omega^{2}\right) \mathrm{d} \omega}{\Delta^{2}+\omega^{2}}
$$

The solution of equation (3.42) was examined in a number of papers (cf. [1-3]), where it was shown that if a self-consistent phonon approximation is applied, then the phase transition becomes of the first order with two characteristic temperatures, one being soft-mode temperature $\tau_{c}$ (when $\Delta^{2}=0$ ), and the other being temperature $\tau_{s}$ at which the structural phase instability occurs (the overheating temperature). From equation (3.42) the soft-mode temperature is estimated as

$$
\tau_{c}^{(1)}=\frac{f_{0}}{3 \mu_{-2}},
$$

where index (1) corresponds to $\sigma^{z}=1$. The constant

$$
\mu_{-2}=\int_{0}^{\infty} \frac{f_{0}}{\omega^{2}} g\left(\omega^{2}\right) \equiv\left\langle\frac{f_{0}}{\omega^{2}}\right\rangle_{\omega},
$$

depending on the type of the cubic lattice, is equal to $1.5-1.3$. For the second characteristic temperature $\tau_{s}$, in the case $f_{0} \ll 1$ one finds the estimate

$$
\tau_{s}^{(1)} \simeq \frac{1}{6}\left(1+f_{0}\right)
$$

Note that the limiting value $\tau_{s} \simeq \frac{1}{6}$, when $f_{0} \rightarrow 0$, is not related to the phase transition, although it has an entirely defined physical meaning: the average kinetic energy of active atoms (i.e. cells) at this temperature is equal to the height of the effective potential barrier, $\frac{3}{2} k_{B} T_{s}=A^{2} / 4 B$. In the case $f_{0} \gg 1$, the estimate of temperature $\tau_{s}$ in the Debye model spectrum is given by 


$$
\tau_{s}^{(1)} \simeq \tau_{c}^{(1)}\left(1+\frac{1}{\omega_{D}^{2}}\right) \simeq \tau_{c}^{(1)}\left(1+\frac{1}{2 f_{0}}\right) .
$$

If $\sigma=0$ the self-consistent equations, the quantities $\Delta_{+}^{2}=\Delta_{-}^{2} \equiv \Delta_{0}^{2}(\tau)$ and $\eta_{+}^{2}=\eta_{-}^{2} \equiv \eta_{0}^{2}(\tau)$ yield

$$
\Delta_{0}^{2}=2 \eta_{0}^{2}-f_{0}=2-3 f_{0}-6 \tau \int_{0}^{\infty} \frac{g\left(\omega^{2}\right) \mathrm{d} \omega^{2}}{\Delta_{0}^{2}+\omega^{2}} .
$$

This equation can be solved only if $f_{0}<\frac{2}{3}$, whereas for $f_{0} \ll 1$ the phase transition is of the same type as follows from equation (3.42). The characteristic soft-mode temperature in this case is estimated as

$$
\tau_{c}^{(0)}=\frac{f_{0}}{3 \mu_{-2}}\left(1-\frac{3}{2} f_{0}\right)=\tau_{c}^{(1)}\left(1-\frac{3}{2} f_{0}\right)
$$

where index (0) corresponds to $\sigma^{z}=0$. Similarly as in the case $\sigma^{z}=1$, one obtains the following estimate for the instability temperature

$$
\tau_{s}^{(0)} \simeq \frac{1}{6}\left(1-2 f_{0}\right)
$$

The estimates obtained show that in the general case of an arbitrary value for parameter $\sigma^{z}$ the temperature $\tau_{c}\left(\sigma^{z}\right)$ falls in the interval within the values (3.43), (3.48), and $\tau_{s}\left(\sigma^{z}\right)$ is determined by relations (3.45), (3.46) and (3.49). Besides, a large hysteresis value $\left(\tau_{s}-\tau_{c}\right) / \tau_{c} \geqslant 1$ corresponds to small values of $f_{0}: f_{0}<0.2$ for $\sigma^{z}=0$, and $f_{0}<0.25$ for $\sigma^{z}=1$; in the case $f_{0} \gg 1$ the hysteresis value is small,

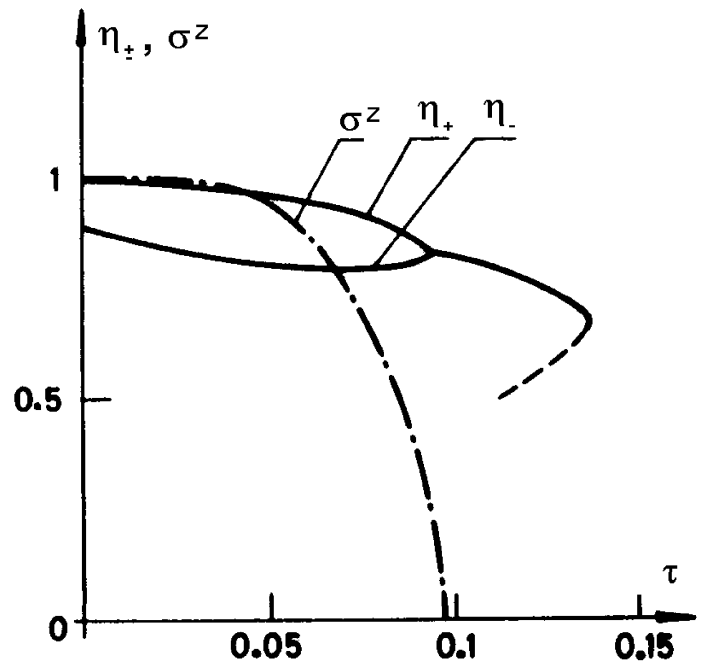

Figure 1. Temperature dependence of the order parameters: $\eta$-average displacement and $\sigma^{z}$-average localization (pseudospin value) for dimensionless parameter $f_{0}=0.10$. in agreement with the estimates based on equation (3.46) and consistent with the results of other authors [15-17] (cf. also [1-3]). It follows from these estimates that the order-disorder phase transition is possible only for small values of $f_{0}$ :

$$
\tau_{k}<\tau_{s}^{(0)}, \quad \text { for } \quad f_{0}<0.12,
$$

if one estimates $\tau_{k} \simeq f_{0}$. At higher values of $f_{0}$ the lattice instability breaks down the order-disorder phase transition, thus giving rise to the displacive phase transition: $\eta_{ \pm}\left(\tau \rightarrow \tau_{s}\right) \rightarrow 0$. At values $f_{0}>0.25$, in agreement with equation (3.12), there is merely one stable atomic equilibrium position at each lattice site, so the displacive-type phase transition is only possible, as described by equation (3.42). 
A mixed-type phase transition, as described by all the three order parameters $\eta_{+}(\tau), \eta_{-}(\tau)$ and $\sigma(\tau)$, may be expected only in a very narrow region:

$$
0.11<f_{0}<0.25
$$

To confirm these general conclusions, the numerical solution of the self-consistent system of equations was obtained for the Debye model frequency spectrum (3.29), $g\left(\omega^{2}\right) \sim \omega, \omega<\omega_{D}$, and the values of the coupling parameter $f_{0}=0.11,0.12$ and 0.15 were taken. The numerical results for $\sigma^{z}(\tau)$ and $\eta_{ \pm}(\tau)$ are presented in figures 1-3. It can be seen that the above estimates are in good agreement with the numerical calculations. The temperature dependence of the spontaneous polarization (3.41) for different $f_{0}$ is shown in figure 4 . Note, that in the region of the order-disorder phase transition $\left(f_{0}<0.11\right)$, as compared with an ordinary Ising model, the spontaneous polarization decreases more rapidly as temperature increases due to the temperature dependence of the effective exchange energy: $\tilde{J}=f_{0} A^{2} / 4 B\left(\eta_{+}+\eta_{-}\right)^{2}$.

We note that these features are also obtained by slightly improved calculations [14] based on the coherent potential approximation developed for the systems with disordered lattices (outlined hereafter in Subsection 3.7 for the case of mixed SPT).

\subsection{The quantum limit in displacive SPT}

In this section we will consider only two cases, namely, a completely ordered $\left(\sigma^{z}=1\right)$ lattice and a completely disordered $\left(\sigma^{z}=0\right)$ one [3]. It is assumed that the right choice of a transverse field $\Omega$ (within IMTF) or Glauber-like relaxational dynamics ensure a transition from $\sigma^{z}=1$ to $\sigma^{z}=0$ at zero temperatures.

\subsubsection{Displacive type phase transition in ordered lattices}

In a completely ordered lattice all the atoms(cells) are in the same state, for example, $\alpha=+1$ and $\sigma^{z}=1$. In this case equation of self-consistency (3.32) takes the following form:

$$
\begin{aligned}
y_{+} & =\frac{\lambda}{2 N} \sum_{q} \frac{1}{\sqrt{\Delta_{+}^{2}+f_{0}-f_{q}}} \\
& =\frac{\lambda}{2} \int_{0}^{\omega_{D}} \frac{g\left(\omega^{2}\right) \mathrm{d} \omega^{2}}{\sqrt{\Delta_{+}^{2}+\omega^{2}}},
\end{aligned}
$$

where the density of the phonon frequencies (3.29) is used.

Taking into account the equilibrium condition (3.12), the self-consistent de-

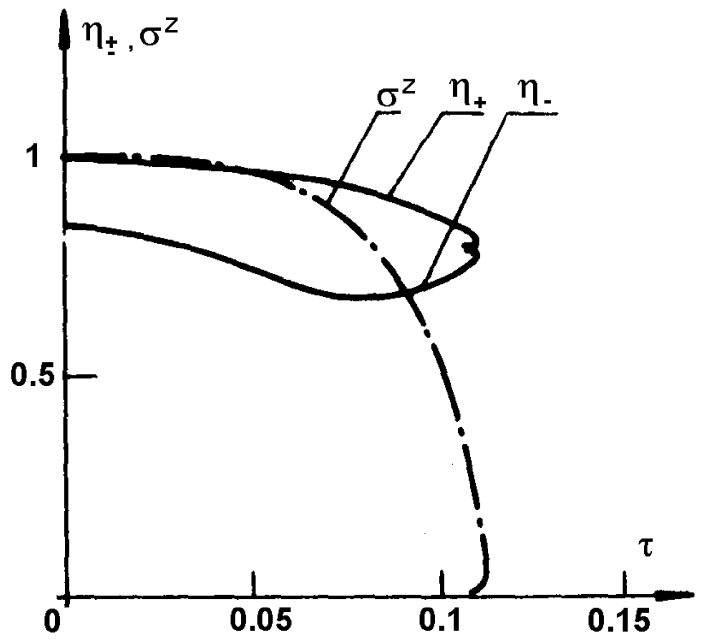

Figure 2. Same as figure 1 , for $f_{0}=0.12$. 
termination of the equilibrium displacement $\eta$ (or the gap in the frequency spectrum $\left.\Delta_{+}^{2}=2 \eta^{2}\right)$ yields:

$$
\eta^{2}=1-\frac{3}{2} \lambda \int_{0}^{\omega_{D}} \frac{g\left(\omega^{2}\right) \mathrm{d} \omega^{2}}{\sqrt{2 \eta^{2}+\omega^{2}}} .
$$

As it can be seen, the solution of this equation for $\eta \neq 0$ exists only if $\lambda<\lambda_{c(1)}$, where the critical value $\lambda_{c(1)}$ is determined by the expression:

$$
\lambda_{c(1)}=\frac{2}{3}\left[\int_{0}^{\omega_{D}} \frac{g\left(\omega^{2}\right) \mathrm{d} \omega^{2}}{\omega^{2}}\right]^{-1}=\frac{2}{3} \frac{\sqrt{f_{0}}}{\mu_{-1}}
$$

here $\mu_{-1}=\overline{\omega^{-1}}$ is the average of the inverse frequency provided that for the Debye spectrum, $g\left(\omega^{2}\right)=3 \omega / \omega_{D}^{3}, \mu_{-1}=3 / 2 \sqrt{2} \simeq 1$, if $\omega_{D}^{2}=2 f_{0}$. Consequently, the displacive type transition in the ordered lattices can take place only if the lattice consists of sufficiently heavy ions, that is if

$$
m>m_{c}=\left(\frac{2}{3} \frac{\sqrt{\varphi_{0}}}{\mu_{-1}} \frac{A}{B}\right)^{-2},
$$

where the critical atomic (cell) mass $\left(m_{c}\right)$ is determined by model parameters (cf. also [15]).

\subsubsection{Displacive type phase transition in disordered lattices}

Let us discuss the effect of disordering on the displacive type phase transition. By putting in equations (3.12) and (3.32) the value $\sigma^{z}=0$ which corresponds to an equal number of atoms in the states $\alpha= \pm 1$ (meaning that $\Delta_{+}^{2}=\Delta_{-}^{2} \equiv \Delta_{0}^{2}$, $y_{+}=y_{-} \equiv y$ and $\left.\eta_{+}=\eta_{-} \equiv \eta\right)$, the following system of equations is then obtained:

$$
\begin{gathered}
\eta^{2}=1-f_{0}-3 y, \\
y=\frac{\lambda}{2} \int_{0}^{\omega_{D}} \frac{g\left(\omega^{2}\right) \mathrm{d} \omega^{2}}{\sqrt{\Delta_{0}^{2}+\omega^{2}}} .
\end{gathered}
$$

Therefore, a self-consistent equation for determining the gap $\Delta_{0}^{2}>0$ takes the form:

$$
\Delta_{0}^{2}=2 \eta^{2}-f_{0}=2-3 f_{0}-3 \lambda \int_{0}^{\omega_{D}} \frac{g\left(\omega^{2}\right) \mathrm{d} \omega^{2}}{\sqrt{\Delta_{0}^{2}+\omega^{2}}}
$$

Hence, the displacive type phase transition $(\eta>0)$ can take place if $\lambda<\lambda_{c(0)}$, where the critical value $\lambda_{c(0)}$ is determined by the condition $\Delta_{0}^{2}\left(\lambda_{c(0)}\right)=0$, wherefrom

$$
\lambda_{c(0)}=\frac{2}{3} \frac{\sqrt{f_{0}}}{\mu_{-1}}\left(1-\frac{3}{2} f_{0}\right)=\lambda_{c(1)}\left(1-\frac{3}{2} f_{0}\right) .
$$

Consequently, the occurence of the lattice disordering decreases both the limiting value of the allowed energy of zero-point fluctuations and the limiting value of the phase transition temperature (3.48). However, it has to be mentioned that the transition into the state $\sigma^{z}=0$ can take place if $f_{0} \ll 1$, only when formula (3.59) is valid. In the case $f_{0}>1$, only the state with $\sigma^{z}=1$ is possible, and formula (3.54) holds. 


\subsection{Phase transition of the mixed type}

As we pointed out in the preceding subsection 3.5, the SPT a of mixed type can occur only in a very narrow region of the reduced lattice parameter $f_{0}(3.51): 0.11<f_{0}<0.25$. Besides various features arising from a considerable

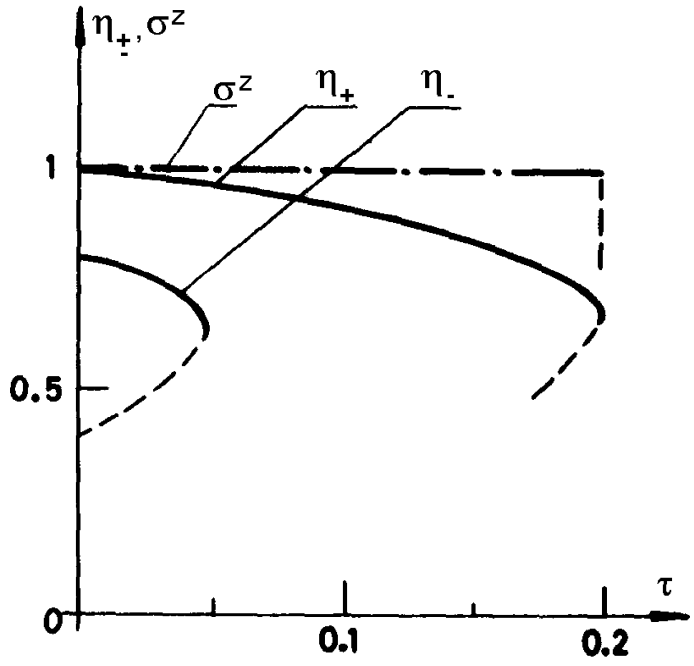

Figure 3. The same as figure 1 , for $f_{0}=$ 0.15 . randomness of equilibrium positions $\eta_{n}$, for this type of SPT of particular interest are the properties of the vibration(phonon)-frequency distribution function. Namely, owing to the temperature dependence (explicit and through the parameter $\sigma_{\alpha}$ ) of randomly distributed quantities $\Delta_{i \alpha}$, the phonon spectrum resembles one of a perfect lattice, not only in a completely ordered phase, $\left(\sigma^{z}=1\right)$, but also in a completely disordered one $\left(\sigma^{z}=0\right)$, as there is no perturbation in this last case $\left(\Delta_{i+}-\Delta_{i-}=0\right)$.

Owing to the disorder of the system, in the case considered a specific approach should be adopted, so that phonons are directly introduced into the Hamiltonian (3.6), i.e. (2.10). Since the disorder is due to single-site random potentials $U\left(\left\{S_{i}^{\alpha}\right\}\right)$, the quasiharmonic form (index $q h$ ) of expression $(2.7), U\left(S_{i}^{\alpha}\right) \approx U_{q h}\left(S_{i}^{\alpha}\right)=A \Delta_{i \alpha}\left(S_{i}^{\alpha}\right)^{2} / 2$, is used in the initial Hamiltonian separated in mutually orthogonal subspaces of eigenstates (3.6), with the gap-parameters $\Delta_{i \alpha}=3\left(\eta_{i \alpha}^{2}+y_{i \alpha}\right)-1 \approx 3\left(\eta_{\alpha}^{2}+y_{\alpha}\right)-1 \equiv \Delta_{\alpha}(3.24)$, $\eta_{i \alpha} \approx \eta_{\alpha}$ (after equation (3.12)), being distributed arbitrarily in each cell. Then,

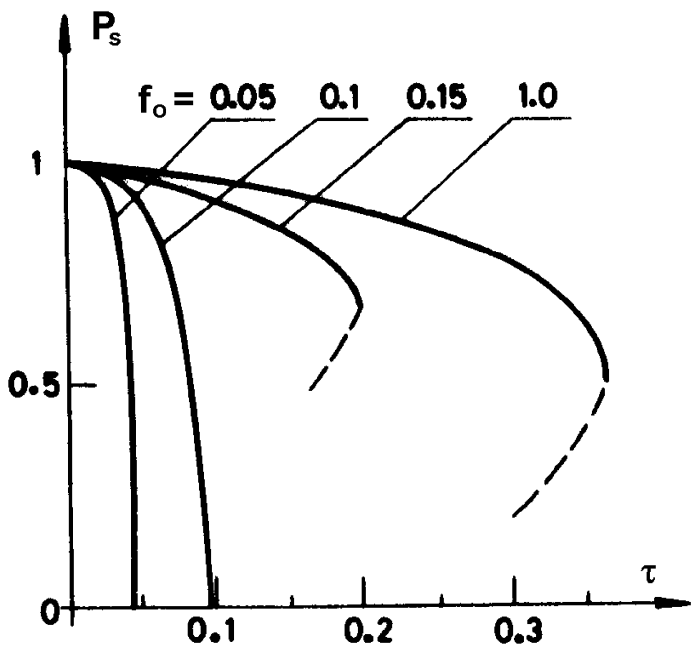

Figure 4. Temperature dependence of the reduced polarization $P_{s}$ for several values of the coupling parameter $f_{0}$. within the framework of the coherentpotential method, by making use of a multiple scattering approach [18] and applying the usual one-site approximation, both the ordinary, $D_{n n^{\prime}}(\omega)$, and the conditional, $D_{n(\alpha) n^{\prime}}(\omega)$, Green functions (but for the given, i.e. fixed configuration $\left\{\sigma_{i}^{\alpha}\right\}$ herein) are found (compare with expressions (3.15), (3.16) together with (3.18), (3.22)). These Green functions permit one to close a self-consistent set of equations for determining the parameters of the effective Hamiltonian which corresponds to substituting potentials $U\left(S_{i}^{\alpha}\right)$ in equation (3.6) by quasiharmonic ones, $U_{q h}\left(S_{i}^{\alpha}\right)$ 
The explicit knowledge of the averaged over all configuration (index $c$ ) Green functions $\left\langle D_{n n^{\prime}}(\omega)\right\rangle_{c}$ and $\left\langle D_{n(\alpha) n^{\prime}}(\omega)\right\rangle_{c}$, as expressed by their Fourier expansions in the reciprocal lattice space, makes possible the calculation for the given values of parameter $\sigma^{z}$ of the lattice phonon spectrum:

$$
\rho\left(\nu^{2}\right)=-(1 / \pi) \operatorname{Im}\left\langle D_{n n}(\nu+\underline{i} \varepsilon)\right\rangle_{c}
$$

and of the phonon density of states for vibrations of an atom(cell) in a fixed state $\alpha$ :

$$
\rho_{\alpha}\left(\nu^{2}\right)=-(1 / \pi) \operatorname{Im}\left\langle D_{n(\alpha), n}(\nu+\underline{i} \varepsilon)\right\rangle_{c} .
$$

In figures 5 and 6 the results of such calculations [14] for the dimensionless coupling constant $f_{0}=0.1$ are presented. In calculating the sum over the reciprocal lattice vectors $q$ in (3.60) and (3.61) the model density of states utilized was of the form (compare with equation (3.29)):

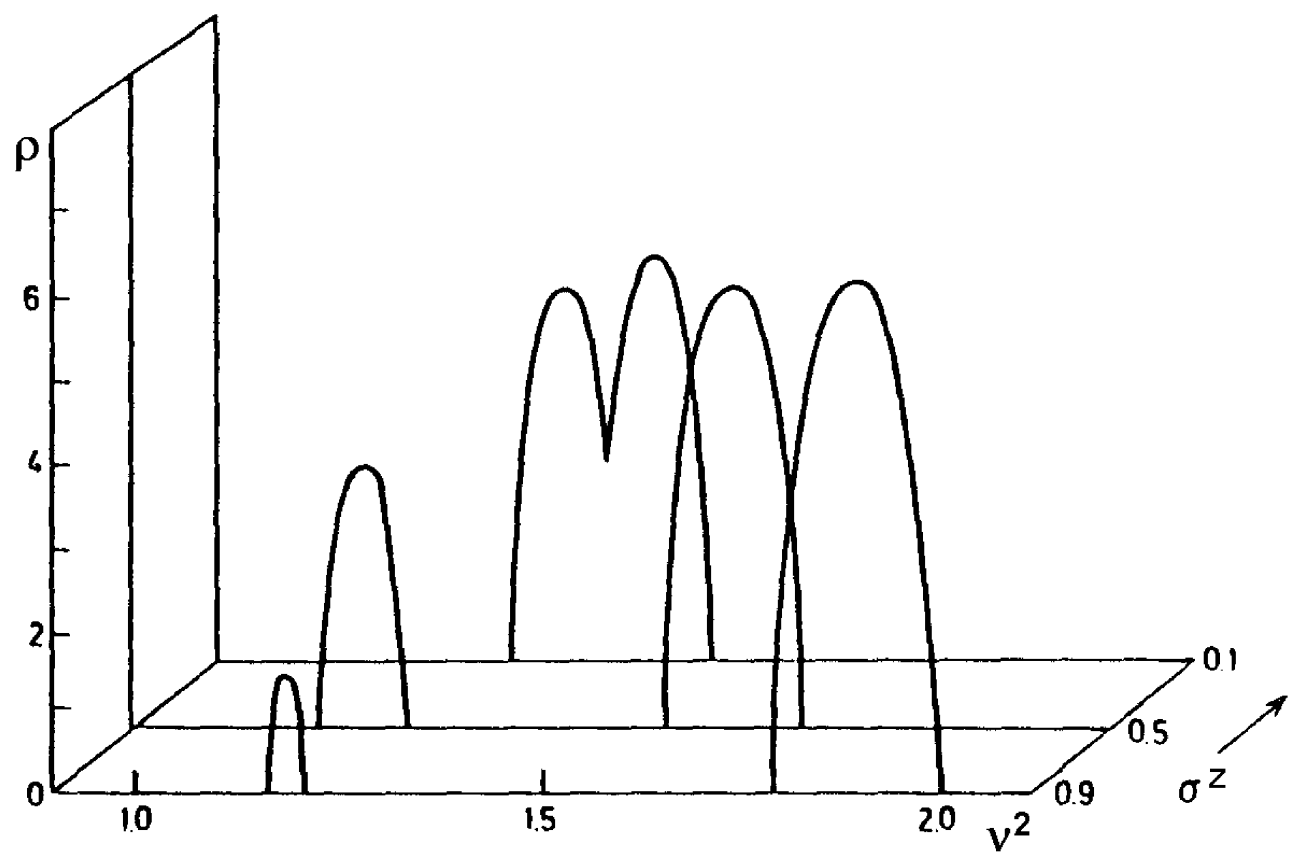

Figure 5. The dependence of the distribution function of the frequencies $\rho\left(\nu^{2}\right)$ on the order parameter $\sigma^{z}$ for $f_{0}=0.1$.

$$
\rho_{0}\left(\nu^{2}\right)=\frac{1}{N} \sum_{q} \delta\left(\nu^{2}-f_{0}+f_{q}\right)=\frac{2}{\pi f_{0}^{2}} \sqrt{\nu^{2}\left(2 f_{0}-\nu^{2}\right)}
$$

for $\nu^{2}<2 f_{0}$. As one can see from figure 5 , the spectrum behaves in different ways when $\sigma^{z} \rightarrow 1\left(\sigma_{+}=1, \sigma_{-}=0\right)$ and when $\sigma^{z} \rightarrow 0\left(\sigma_{+}=\sigma_{-}=1 / 2\right):$ in the first case, when all the particles are ordered in the state $(+)$, the band corresponding to the state (-) vanishes, while, as $\sigma^{z} \rightarrow 0$, the both bands merge into one. It should be noted that the calculations of the phonon spectrum in order-disorder SPTs are usually missing, and, therefore, the experimental verification of peculiar spectral features, as pointed in figures 5 and 6 , is of particular interest. 


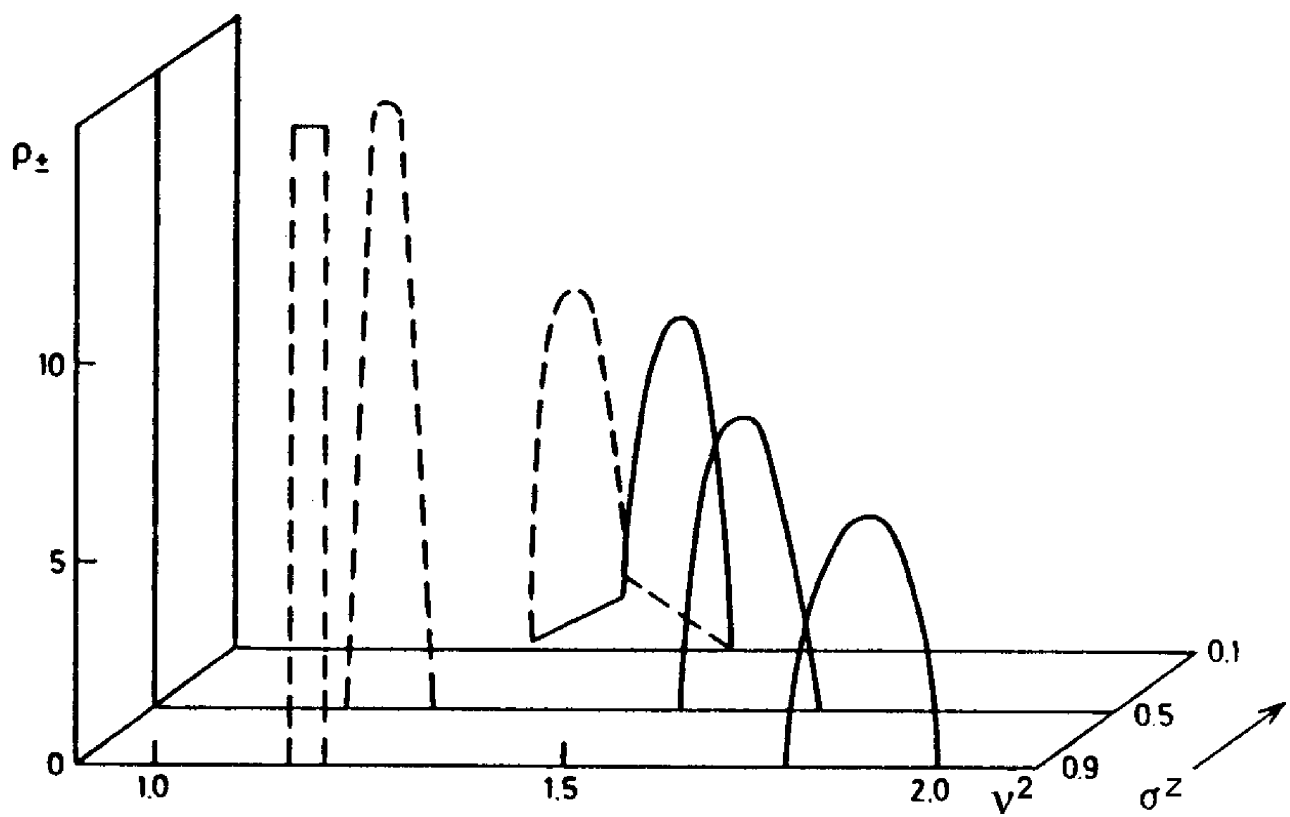

Figure 6. The dependence of the vibration-frequency distribution function on the order parameter $\sigma^{z}$ in the state $\alpha= \pm$ for $f_{0}=0.1$ : the solid line represents $\rho_{+}$, the dash line represents $\rho_{-}$.

\section{Generalized model of SPT: ordering, tunelling and phonons}

\subsection{A need for the incorporation of the atomic tunnelling motion}

The previous studies of rather simple models [1-4] have shown that both the displacive and order-disorder type excitations play an essential role in the dynamics of SPTs. At the same time a considerable effort has also been made to describe the corresponding both types of SPTs (ferroelectric ones included) within a single universal model (cf.[5]). However, the single-particle tunnelling motion of active atoms has not been explicitly taken into account. The incorporation of the tunnelling motion as an additional degree of freedom leads to collective excitations which may have a soft mode character [5] or cause the appearance of a central peak $[5,6]$. Since the tunnelling energies (of the order of the ground state quantum splitting, $\Omega$ ) are usually much smaller than the characteristic phonon energies, the role of such excitations is predominant at low temperatures $\left(k_{\mathrm{B}} T \sim \Omega\right)$ in which case, the SPT can be viewed as a transition that is predominantly of the order-disorder type. On the other hand, in addition to the renormalization of pseudospin-energy parameters of the well-known De Gennes type, the higher phonon excitations can lead to the SPT of the displacive type (against a certain vibrational mode) at higher temperatures.

In this section an interpolated way for improving the unified analytical procedure in the SPT dynamics is presented. For this purpose we take into account selfconsistently (within the Bogolyubov variational approach) excitations of the both 
types (displacive and order-disorder) - in order to comprise both the tunnelling and higher phonon oscillations of active atoms (i.e. cells) within the framework of a hybridized pseudospin-phonon model Hamiltonian separated in the corresponding variables. This is achieved by representing the cooperative atomic motion as a slow tunnelling process among several (in the simplest case, two) equilibrium positions in addition to familiar phonon-like oscillations around some momentary rest position. On the basis of the self-consistent phonon and molecular-field approximations a complete system of coupled equations for two order parameters (average displacement $\eta \sim r_{s a}$ and average localization $\sigma^{z}$ ) is obtained. Both qualitative and detailed numerical analyses show that the SPT (of the first or second order) can be either of an order-disorder, a displacive or of a mixed type, depending predominantly on the ratio of a two-particle potential to a single-particle one and, in a lesser degree, on the ratio of the zero-point vibrational energy to the height of a single-particle potential barrier. The possible SPT of the both types at zero temperature (quantum limit), as well as the relation between these results and other relevant treatments are also discussed.

\subsection{The model phonon-pseudospin Hamiltonian}

Our previous $[1,2]$ coordinate representation $s_{i}=\sum_{\alpha} \sigma_{i \alpha}\left(b_{i \alpha}+u_{i \alpha}\right)(3.3)$ described the random atomic (cell) distribution (within the Ising model through the projection operator $\left.\sigma_{i \alpha}=1 / 2\left(1+\alpha \sigma_{i}^{z}\right)\right)$, over two $(\alpha=+,-)$ equilibrium positions $\left(b_{i \alpha}\right)$ at every site $i$, as well as thermal atomic fluctuations $\left(u_{i \alpha}\right)$ around one or another momentary rest positions $\left(b_{i \alpha}\right)$ ("left" and "right" phonons) (see Subsection 3.2 ). However, to elucidate more profoundly such an additional pseudospin degree of freedom $\left(\sigma_{i}^{z}\right)$, one has to take into account the inherent quantum mechanical effect manifested in a single-particle tunnelling motion of active atoms inside some (real or effective) double-well potential, which was fully missing in our previous investigations and rather implicit in the approaches of other authors (cf. $[1,2]$ and Refs. therein). For this purpose we suggested [4] the "left-right" representation of the model Hamiltonian (3.6) in the non-orthogonal pseudospin (i.e. "left"- "right") basis. However, in accordance with the exhaustive analyses of many authors (cf. [5]), a clearer physical picture (and a rather transparent procedure) can be introduced by natural generalization of the traditional concept of atomic equilibrium states. Thus, a time dependent local normal coordinate may be decomposed into a slow tunnelling-like displacement $\left(r_{i}\right)$ and comparatively fast superimposed deviations of the phonon type $\left(u_{i}\right)$ [5-7] (compare with (3.1)):

$$
s_{i}=r_{i}+u_{i} ; \quad\left\langle u_{i}\right\rangle=0 .
$$

Such a representation holds under the "adiabatic" condition $\Omega \ll \omega_{0}$, $\omega_{0}$, being a characteristic frequency of lattice vibrations.

Having inserted definition (4.1) into the general Hamiltonian (2.4) (assuming one-component coordinate $R_{i} \equiv x_{i}$ and momentum $\left.P_{i}=-i \hbar \frac{\partial}{\partial R_{i}} \equiv-i \hbar \frac{\partial}{\partial x_{i}}\right)$ it can be written in a trial form separated in the corresponding variables: 


$$
H_{0}=H_{p h}\left(\left\{u_{i}\right\}\right)+H_{t}\left(\left\{r_{i}\right\}\right),
$$

where the phonon-like (index $p h$ ) and tunnelling-like (index $t$ ) Hamiltonians are given by

$$
\begin{gathered}
H_{p h}=\sum_{i} \frac{P_{i}^{2}}{2 m}+\frac{1}{2} \sum_{i j} \phi_{i j} u_{i} u_{j}, \\
H_{t}=\sum_{i}\left[\frac{p_{i}^{2}}{2 m}+\tilde{U}\left(r_{i}\right)\right]+\frac{1}{2} \sum_{i \neq j} C_{i j}\left(r_{i}-r_{j}\right)^{2} ;
\end{gathered}
$$

$\phi_{i j}, C_{i j}$ and $\tilde{U}\left(r_{i}\right)$ are variational parameters and $P_{i}$ and $p_{i}$ - canonical conjugate momenta to $u_{i}$ and $r_{i}$, respectively. For a strongly anharmonic motion described by equation (4.4) it is convenient to introduce the energy representation comprising single-particle ground doublet (symmetric $\left(\psi_{s}\right)$ and antisymmetric $\left(\psi_{a}\right)$ ) states which satisfy the eigenvalue equation:

$$
\left[\frac{P_{i}^{2}}{2 m}+\tilde{U}\left(r_{i}\right)\right] \psi_{s, a}\left(r_{i}\right)=\varepsilon_{s, a} \psi_{s, a}\left(r_{i}\right) .
$$

Going over to the pseudospin representation, $H_{t}(4.4)$ is presented in the wellknown De Gennes form (e.g. IMTF) (cf. [5]):

$$
H_{s}=-\Omega \sum_{i} \sigma_{i}^{x}-\frac{1}{2} \sum_{i} J_{i j} \sigma_{i}^{z} \sigma_{i}^{z}+E_{0},
$$

where the energy parameters $\Omega, J_{i j}$ and $E_{o}$ are simple functions of $\varepsilon_{\alpha}, C_{i j}$, and the matrix elements $r_{\alpha \beta}=\left\langle\alpha\left|r_{i}\right| \beta\right\rangle$ and $r_{\alpha \alpha}^{2}=\left\langle\alpha\left|r_{i}^{2}\right| \alpha\right\rangle(\alpha, \beta=s, a)$ calculated with the wave functions $\psi_{\alpha}$ in equation (4.5):

$$
\begin{array}{r}
\Omega=\frac{1}{2}\left(\varepsilon_{a}-\varepsilon_{s}\right)+r_{-}^{2} C_{0}, \\
E_{0}=\frac{1}{2}\left(\varepsilon_{a}+\varepsilon_{s}\right)+r_{+}^{2} C_{0}, \\
J_{i j}=2 r_{s a}^{2} C_{i j} ; \\
r_{ \pm}^{2}=\frac{1}{2}\left(r_{a a}^{2} \pm r_{s s}^{2}\right) ; \\
C_{0}=\sum_{j} C_{i j} .
\end{array}
$$

The variational parameters $\phi_{i j}, C_{i j}$ and $\tilde{U}\left(r_{i}\right)$ are determined from the Bogolyubov variational principle for the free energy of system $F$, namely, from the condition of stationarity of free energy:

$$
F_{1}=F_{0}+\left\langle H-H_{0}\right\rangle_{0} \geqslant F
$$


with respect to variations over these parameters or, equivalently, over the corresponding correlation functions (cf. [8]). In the above expression (4.8) symbol $<\ldots\rangle_{0}$ stands for the statistical averaging with the trial Hamiltonian $H_{0}((4.2)-$ $(4.7)$ ), to which free energy $F_{0}$ corresponds.

Computing free energy $F_{1}(4.8)$ where

$$
\begin{gathered}
F_{0}=-k_{\mathrm{B}} T \ln \operatorname{Sp}\left\{e^{-H_{0} / T}\right\} \\
\left\langle H-H_{0}\right\rangle_{0}=\operatorname{Sp}\left\{e^{\frac{F_{0}-H_{0}}{T}}\left(H-H_{0}\right)\right\}= \\
=\sum_{i}\left\langle U\left(r_{i}+u_{i}\right)\right\rangle_{0}+\frac{1}{2} \sum_{i j}\left\langle V\left(r_{i}+u_{i}, r_{j}+u_{j}\right)\right\rangle_{0}- \\
-\sum_{i}\left\langle\tilde{U}\left(r_{i}\right)\right\rangle_{0}-\frac{1}{2} \sum_{i j} \phi_{i j}\left\langle u_{i} u_{j}\right\rangle_{0}-\frac{1}{2} \sum_{i j} C_{i j}\left\langle\left(r_{i}-r_{j}\right)^{2}\right\rangle_{0}
\end{gathered}
$$

under the assumption that condition $\left\langle P_{i} p_{i}\right\rangle_{0}=0$ holds, for the variational parameters of the trial Hamiltonians (4.3) and (4.4) (i.e. (4.6)) the following equations are obtained:

$$
\begin{gathered}
\phi_{i j}=\frac{\delta}{\delta\left\langle u_{i} u_{j}\right\rangle_{0}}\left\{2 \sum_{i}\left\langle U\left(r_{i}+u_{i}\right)\right\rangle_{0}+\sum_{i \neq j}\left\langle V\left(r_{i}+u_{i}, r_{j}+u_{j}\right)\right\rangle_{0}\right\}, \\
2 r_{s a}^{2} C_{i j}=\frac{\delta}{\delta\left\langle\sigma_{i}^{z} \sigma_{i}^{z}\right\rangle_{0}}\left\langle V\left(r_{i}+u_{i}, r_{j}+u_{j}\right)\right\rangle_{0},
\end{gathered}
$$

and

$$
\begin{aligned}
& \frac{\delta}{\delta\left\langle\sigma_{i}^{x}\right\rangle_{0}} \sum_{i}\left\langle\tilde{U}\left(r_{i}\right)\right\rangle_{0}=\frac{\delta}{\delta<\sigma_{i}^{x}>_{0}}\left\{\sum_{i}\left\langle U\left(r_{i}+u_{i}\right)\right\rangle_{0}+\right. \\
& \left.+\frac{1}{2} \sum_{i j}\left\langle V\left(r_{i}+u_{i}, r_{j}+u_{j}\right)\right\rangle_{0}-\frac{1}{2} \sum_{i j} C_{i j}\left\langle r_{i}^{2}+r_{j}^{2}\right\rangle_{0}\right\} .
\end{aligned}
$$

The self-consistent system of equations (4.3)-(4.7), (4.11)-(4.13) determines the phase transition of the model and describes the mutual influence of phonon and pseudospin subsystems if the potentials $U\left(s_{i}\right)$ and $V\left(s_{i}, s_{j}\right)$ in equation $(2.4)$ are appropriately modelled.

\subsection{The structural phase transition in the model}

Having chosen a single-particle double-well potential $U\left(s_{i}\right)$ in the convenient form $(2.7)$ and a pair-potential $V\left(s_{i}, s_{j}\right)$ in the familiar harmonic approximation (2.8), we assume the model Hamiltonian of the system in the previous form (2.10): 


$$
H=\sum_{i}\left(-\frac{\hbar^{2}}{2 m} \frac{\partial^{2}}{\partial s_{i}^{2}}-\frac{A}{2} s_{i}^{2}+\frac{B}{4} s_{i}^{4}\right)+\frac{1}{4} \sum_{i j} \varphi_{i j}\left(s_{i}-s_{j}\right)^{2} .
$$

The variational approach for this Hamiltonian yields:

$$
\phi_{i j}=\delta_{i j}\left(A \Delta+\varphi_{0}\right)-\varphi_{i j}\left(1-\delta_{i j}\right) ; \quad 2 C_{i j}=\varphi_{i j}, \quad \varphi_{0}=\sum_{j} \varphi_{i j}
$$

where

$$
\Delta=3(B / A)<r_{i}^{2}>_{0}-a, a=1-3(B / A)\left\langle u_{i}^{2}\right\rangle .
$$

The effective (renormalised) single-particle (cell) potential in (4.4) can be written in the form:

$$
\tilde{U}\left(r_{i}\right)=-\frac{\tilde{A}}{2} r_{i}^{2}+\frac{B}{4} r_{i}^{4} ; \quad \tilde{A}=A-3 B\left\langle u_{i}^{2}\right\rangle_{0} \equiv A a
$$

The self-correlation displacement functions relevant to the nature of the SPT are determined by the following approximative equations (of the RPA type):

$$
\begin{gathered}
\left\langle u_{i}^{2}\right\rangle_{0}=\frac{1}{N m} \sum_{k} \frac{1}{2 \omega_{k}} \operatorname{coth} \frac{\omega_{k}}{2 k_{\mathrm{B}} T}, \\
\left\langle r_{i}^{2}\right\rangle_{0}=\frac{1}{2}\left[\left(r_{s s}^{2}+r_{a a}^{2}\right)+\left(r_{s s}^{2}-r_{a a}^{2}\right)+\left(r_{s s}^{2}+r_{a a}^{2}\right)\left\langle\sigma_{i}^{x}\right\rangle_{0}\right],
\end{gathered}
$$

where the phonon frequency $\omega_{k}$ is given by the equation

$$
m \omega_{k}^{2}=A \Delta+\varphi_{0}-\varphi_{k} ; \quad \varphi_{k}=\sum_{j} \varphi_{i j} e^{i k\left(l_{i}-l_{j}\right)}
$$

In the mean(molecular)-field approximation (equivalent to RPA for pseudospins) (see, e.g., [19]) one obtains:

$$
\begin{gathered}
\left\langle\sigma_{i}^{x, z}\right\rangle=\sigma^{x, z}=\frac{h_{x, z}}{h} \tanh \frac{h}{k_{\mathrm{B}} T} \\
h_{x}=\Omega, \quad h_{z}=J_{0} \sigma^{z}, \quad h^{2}=h_{x}^{2}+h_{z}^{2} ; \quad J_{0}=\sum_{j} J_{i j}=r_{s a}^{2} \varphi_{0} .
\end{gathered}
$$

Thus, the SPT-system is described by the solution of the above self-consistent system of equations which, owing to equation (4.5), can be obtained only numerically. Nevertheless, a qualitative analysis for the limiting cases is possible. 


\section{a) Order-disorder transition}

Analogously to the analysis in the previous sections, in the weak coupling limit of small $\varphi_{0}$, i.e. in the temperature region when

$$
\varphi_{0} \ll \tilde{A}=A-3 B\left\langle u_{i}^{2}\right\rangle_{0},
$$

the order-disorder transition is possible in the pseudospin subsystem as characterized by the order parameter $\sigma^{z}$. In the molecular field approximation for the order-disorder transition temperature $T_{c}$ one finds:

$$
T_{c} \simeq J_{0} \frac{2 q}{\ln \frac{1+q}{1-q}} ; \quad q=\Omega / J_{0} \leq 1 .
$$

The estimates obtained in the case of weak tunnelling $\left(\Omega \ll J_{0} ; r_{s s}^{2} \sim r_{a a}^{2} \sim\right.$ $\left.r_{s a}^{2} \sim \tilde{r}_{0}^{2}=\tilde{A} / B\right)$ correspond to the results of section 3 (for $f_{0} \ll 1$, therein), namely,

$$
T_{c} \sim J_{0} \sim \varphi_{0} \tilde{r}_{0}^{2} \sim \varphi_{0} \frac{\tilde{A}}{B} \ll U_{0} .
$$

Note that phonon excitations do not play an essential role in this case, since $\left\langle u_{i}^{2}\right\rangle_{0} \ll \tilde{r}_{0}^{2}$.

\section{b) Displacive transition}

When the temperature is raised, the atomic fluctuations $\left\langle u_{i}^{2}\right\rangle_{0}$ cannot be neglected and the character of the coupling could be changed, i.e. $\varphi_{0} \gg \tilde{A}$ (even for $\left.\varphi_{0} \ll A\right)$, thus, leading to the displacive phase transition: $\Delta\left(T_{0}\right) \rightarrow 0$ and $\tilde{r}_{0}^{2}\left(T_{0}\right) \rightarrow$ 0 . In the classical limit of high temperatures $\left(T \gtrsim T_{0}>T_{c}\right)$ [1-7],

$$
\tilde{A}\left(T_{0}\right)=0, \quad\left\langle u_{i}^{2}\right\rangle_{0}=\frac{1}{3} \frac{A}{B},
$$

while in the strong-coupling limit $\left\langle u_{i}^{2}\right\rangle \sim T / m \omega_{0}^{2} \sim T / \varphi_{0}$ also holds and the displacive transition temperature is estimated as

$$
T_{0} \sim \frac{1}{3} \varphi_{0}(A / B)
$$

provided that for the model considered the inequality $T_{c} \sim \varphi_{0} \tilde{A} / B<T_{0} \sim \varphi_{0} A / B$ is constantly valid. For both limiting types of SPTs the similar results have been obtained in subsection 3.5.

\subsection{Approximation of a double well by two truncated harmonic oscillators}

For the trial wave functions in equation (4.5) one can assume the linear combinations of the ground states, $\psi_{0}^{-}$and $\psi_{0}^{+}$, referred to the "left" $(-)$and "right" $(+)$ unperturbed harmonic oscillators, respectively, to be of the form: 


$$
\psi_{s, a}=[2(1 \pm \rho)]^{-1 / 2}\left[\psi_{0}^{+}(r) \pm \psi_{0}^{-}(r)\right]
$$

where

$$
\begin{array}{r}
\psi_{0}^{ \pm}(r)=\psi_{0}\left(r \pm \tilde{r}_{0}\right) ; \quad \psi_{0}(r)=\left(a_{0} \sqrt{\pi}\right)^{-1 / 2} \exp \left(-r^{2} / 2 a_{0}^{2}\right) \\
a_{0}^{2}=\hbar / m \bar{\omega} ; \quad \bar{\omega}^{2}=\tilde{k} / m, \tilde{r}_{0}=(\tilde{A} / B)^{1 / 2}
\end{array}
$$

Here $\rho$ is the overlap integral of the "left" and "right" states; the harmonic forceconstant $k=2 A$ is renormalised to be $\tilde{k}=2 \tilde{A}$ (in the approximation of a strong particle (i.e. cell) localization). By performing the corresponding calculations one finds:

$$
\begin{gathered}
\rho=\int \psi_{0}^{+}(r) \psi_{0}^{-}(r) d r=\exp \left\{-\frac{\tilde{A}^{2} / B}{\bar{\omega} / 2}\right\}=\exp (-1 / \lambda) ; \\
\lambda=\frac{\bar{\omega}}{2 \tilde{A}^{2} / B}=a_{0}^{2} / \tilde{r}_{0}^{2}=\frac{\lambda_{0}}{\sqrt{2}(1-3 y)^{3 / 2}},
\end{gathered}
$$

where the temperature independent quantum parameter (compare with equation (2.16)) characterizing the zero-point vibrations $\lambda_{0}=\hbar \omega_{0} /\left(A^{2} / B\right), \omega_{0}=\sqrt{A / m}$ is introduced, and parameter $y=(B / A)\left\langle u_{i}^{2}\right\rangle$ is the reduced average quadratic "fast" displacement ( after equation (4.18)). Consequently, for the parameters of the pseudospin Hamiltonian (4.6) the following expressions are obtained:

$$
\begin{gathered}
\Omega=\frac{A^{2}}{4 \sqrt{2} B} \frac{\lambda_{0} \rho \sqrt{1-3 y}}{1-\rho^{2}}\left(1+\frac{3}{\lambda}\right), \\
r_{s a}^{2}=\tilde{r}_{0}^{2} /\left(1-\rho^{2}\right) ; \quad \tilde{r}_{0}^{2}=(A / B)(1-3 y), \\
r_{s s}^{2}=\frac{\tilde{r}_{0}^{2}}{1 \pm \rho}\left[1+\frac{\lambda}{2}(1 \pm \rho)\right],
\end{gathered}
$$

where the reduced average quadratic "slow" displacement (4.19) becomes

$$
\bar{r} \equiv \frac{B}{A}\left\langle r_{i}^{2}\right\rangle_{0}=\frac{1-3 y}{1-\rho^{2}} \quad\left[1+\frac{\lambda\left(1-\rho^{2}\right)}{2}-\rho \sigma^{x}\right] .
$$

To make explicit the reduced average quadratic "fast" displacement (4.18), the spectral density of phonon frequencies is introduced:

$$
g(\nu)=\frac{2}{\pi} \sqrt{1-\nu^{2}} ; \quad \nu=\frac{\omega}{A / m}
$$

and then one finds

$$
y=\lambda_{0} \int_{-1}^{1} \frac{\mathrm{d} \nu g(\nu)}{2 \sqrt{\Delta-f_{0} \nu}} \operatorname{coth} \frac{\lambda_{0} \sqrt{\Delta-f_{0} \nu}}{2 \theta}, \quad f_{0}=\varphi_{0} / A ; \quad \theta=\frac{k_{\mathrm{B}} T}{A^{2} / B}
$$

where 


$$
\Delta=3(y+\bar{r})-1
$$

is a gap in the phonon spectrum (4.20).

Finally, owing to equation (4.32), the spontaneous polarization (per cell) of the system is simply expressed as a product of the displacive-like $(\eta)$ and the order-disorder-like $\left(\sigma^{z}\right)$ order parameters:

$$
P_{s}=\frac{1}{N} \sum_{i}\left(\frac{B}{A}\right)^{1 / 2}\left\langle r_{i}\right\rangle_{0}=\left(\frac{B}{A}\right)^{1 / 2} r_{s a} \sigma^{z}=\eta \sigma^{z},
$$

where the order parameters $\sigma^{z}$ and $\eta$ are given by the above self-consistent procedure: $\sigma^{z}$ - by equation (4.21) and $\eta \equiv(B / A)^{1 / 2} r_{s a}$ - by the expression

$$
\eta=\left\{\frac{1-3 y}{1-\rho^{2}}\right\}^{1 / 2} .
$$

Both the "order-disorder" $\left(\sigma^{z}\right)$ and the "displacive" $(\eta)$ order parameters are to be found as a self-consistent solution of equations (4.21), (4.29)-(4.37). For a given set of the reduced energy parameters $\left(\lambda_{0}, f_{0}\right)$ the competition of these order parameters determines the character of the SPT.

\subsection{The quantum limit of zero temperature}

At zero temperature equations (4.21) and (4.36) become

$$
\begin{gathered}
\sigma^{z}=\frac{1}{J_{0}} \sqrt{J_{0}^{2}-\Omega^{2}}, \quad \sigma^{x}=\frac{\Omega}{J_{o}} ; \\
y=\lambda_{0} \int_{-1}^{1} \frac{\mathrm{d} \nu \sqrt{1-\nu^{2}}}{\pi \sqrt{\Delta-f_{0} \nu}} .
\end{gathered}
$$

As it is easily seen from equation (4.40), $\sigma^{z} \geqslant 0$ if $\Omega \leqslant J_{0}$. Using equation (4.41) with equations $(4.21),(4.32)$, one obtains the condition for $\lambda_{0}$,

$$
\frac{\rho_{c}}{4 f_{0}}\left(3+\lambda_{0}\right)\left(1-3 y_{c}\right)=1
$$

which defines the maximum $\lambda_{0}\left(\lambda_{0}^{c}\right)$ at which $\sigma^{z}>0$ is still possible. In a simplified case, when $y_{c} \ll \frac{1}{3}$, the graphical solution of equation (4.42) gives

$$
\lambda_{0}^{c} \simeq 1 / \ln \left[(3 / 4) f_{0}\right] .
$$

Hence, if $\lambda_{0}>\lambda_{0}^{c}$, then $\sigma^{z}=0$, even at zero temperature.

The order parameter $\eta$ can also vanish at zero temperature. Using equations (4.36), (4.37) and (4.39), one obtains

$$
\lambda_{0}^{p h} \simeq 2 \sqrt{f_{0}} .
$$


In such a way the zero-point vibrations can destroy the ordered ground state at $T=0 \mathrm{~K}$, both in the pseudospin and the phonon subsystems. One can expect that $P_{s}$ vanishes either in $\sigma^{z}$ or in $\eta$, depending on the competition between $\lambda_{0}^{c}$ and $\lambda_{0}^{p h}$, i.e. on the lesser of the two. The corresponding estimates of $\lambda_{0}^{c}$ and $\lambda_{0}^{p h}$ for various values of parameter $f_{0}$ are given in table $\mathrm{I}$.

TABLE I

\begin{tabular}{c|cccc}
\hline$f_{0}$ & 0.05 & 0.10 & 0.30 & 0.50 \\
$\lambda_{0}^{c}$ & 0.37 & 0.50 & 1.95 & 2.50 \\
$\lambda_{0}^{p h}$ & 0.44 & 0.62 & 1.10 & 1.40
\end{tabular}

\subsection{The numerical results}

\subsubsection{The case of the double-well potential modelled by two harmonic os- cillators}

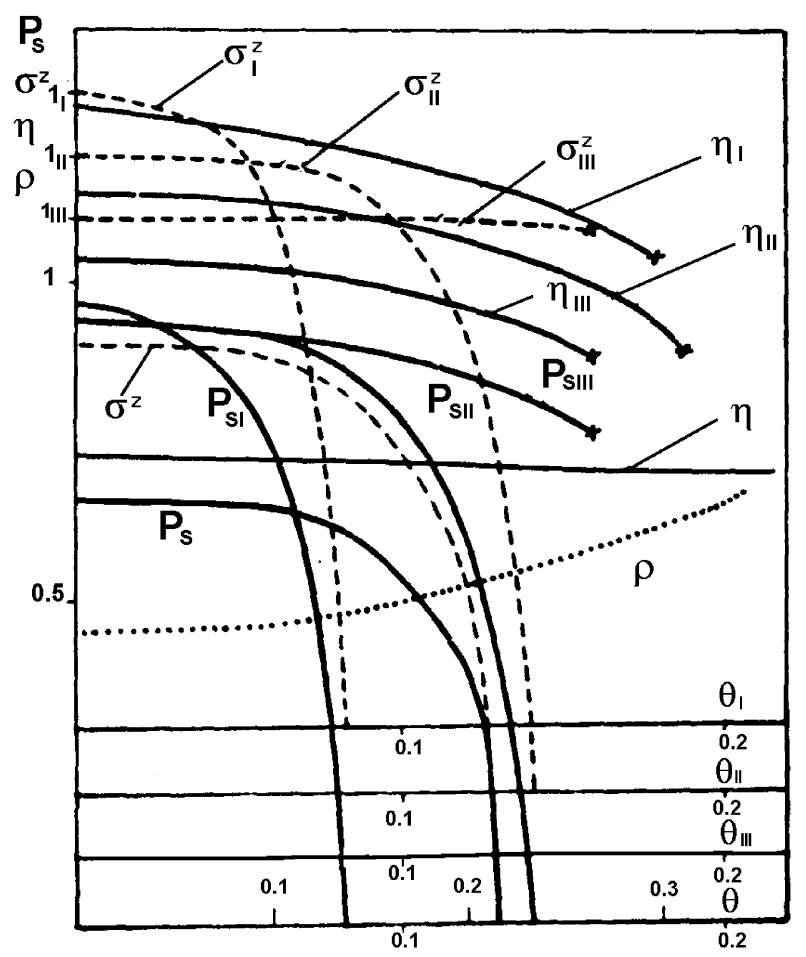

Figure 7. The order parameters sigma ${ }^{z}$ and $\eta$, the polarization $P_{s}$ and the overlapping parameter $\rho$ as functions of temperature $(\theta)$. Parameters $\sigma^{z}, \eta, P_{s}$-upon $\theta$-scale and $\rho$-upon above-scaled $\theta$-abscissa correspond to $\lambda_{0}=$ 0.5 and $f_{0}=0.5$; the marked parameters $\sigma_{I}^{z}$, $\eta_{I}, P_{s}^{I}$, etc., upon $\theta_{I}$-scale, etc., correspond to $\lambda_{0}=0.1$ and various $f_{0}=0.1,0.2,0.6$, respectively. parameter (here $\eta$ ) and the exact curves for $\sigma^{z}$ [15].
The system of self-consistent equations (4.21) and (4.29)(4.39) is solved numerically and $\sigma^{z}(\theta), \eta(\theta), P_{s}(\theta)$ (for various $f_{0}$ and $\lambda_{0}$ ) and $\rho(\theta)$ (for $f_{0}=\lambda_{0}=$ 0.5 as well as $\sigma^{z}\left(\lambda_{0}\right), \eta\left(\lambda_{0}\right)$ (for $f_{0}=0.6$ and $\left.\theta=0.001\right)$ and $\rho\left(\lambda_{0}\right)$ (for various $f_{0}=0.1,0.2,0.6$ and $\theta=0.001)$ are presented in figures 7 and 8 , respectively [5]. It can be observed that the qualitative estimates in the preceding subsections (4.3-4.5) are in good agreement with the numerical calculations. For all marked parameters (I to III) the overlapping integral $\rho$, as calculated, is of the order 0.003 at $T \simeq T_{S P T}$. The corresponding curves in figure 7 are in agreement with our previous ones [1] (cf. section 3). In the weakcoupling limit $f_{0}=f_{0}^{1} /\left(1-f_{0}^{1}\right) \ll$ 1 ( $f_{0}^{1}$ being the reduced coupling constant $f_{0}$ in [1], i.e. in equation (3.13)), the proper account of tunnelling effects is taken for $\rho \ll 1$ (at least $\rho<0.5$ and $\lambda_{0}<0.5$ ), in agreement with the previous results for both the order 
The appearance of imaginary solutions in both figures, 7 and 8, is assigned by cross-points. A discontinuity itself in the displacive SPT (but in the case $f_{0} \ll 1$ ) is a well-known characteristic of the self-consistent phonon approximation (cf. $[1,7]$ ). In the strongcoupling limit $\left(f_{0} \gg 1\right)$ (when the tunnelling can be disregarded) the displacive SPT does occur and is properly described as previously [1]. As it can be observed in figure 7 , the case "III" $\left(f_{0}=\right.$ $0.6)$ corresponds to a mixed SPT. In figure $8 \eta\left(\lambda_{0}\right)$ has a minimum indicated as $\lambda_{0}^{p h(1)} ; \lambda_{0}^{p h(2)}$ denotes a value at which $\eta$ becomes imaginary and $\lambda_{0}^{c}$ is a critical $\lambda_{0}$ for the pseudospin subsystem (for $\left.\lambda_{0}>\lambda_{0}^{c}, \sigma^{z}=0\right)$.

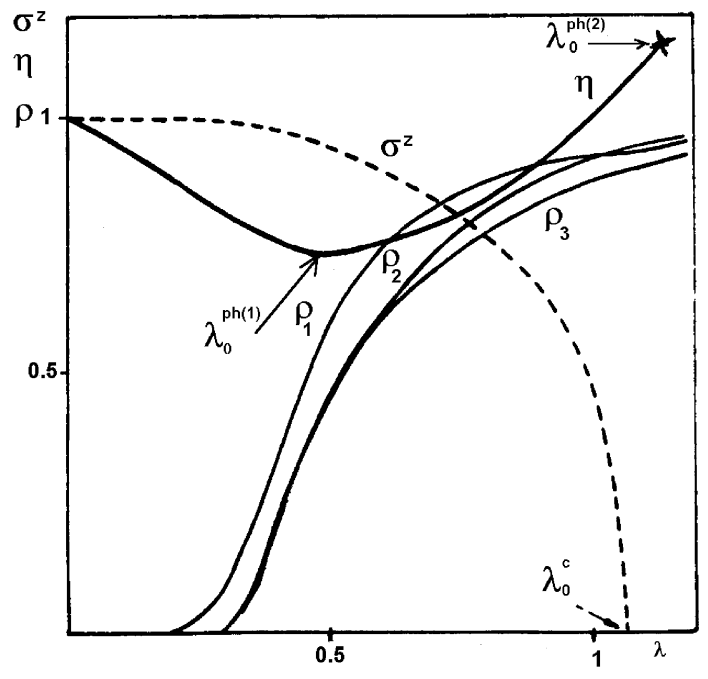

Figure 8. The order parameters $\sigma^{z}$, $\eta$ and the overlapping parameter $\rho$ as functions of the quantum parameter $\lambda_{0}$. $\sigma^{z}$ and $\eta$ are presented for $f_{0}=0.6$; $\rho_{1}, \rho_{2}, \rho_{3}$ correspond to various $f_{0}=$ $0.1,0.6,1.0$, respectively.

\subsubsection{The exact results based on the model of quartic oscillator}

The self-consistent numerical calculations using the renormalized double-well potential (4.17) are also performed, provided that the overlap of the exact (index exc) wave functions $\psi_{s}$ and $\psi_{a}, \rho_{\text {exc }}=\langle s \mid a\rangle_{\text {exc }}$, is now equal to zero [5]. The dependence of all the relevant parameters for various $f_{0}, \lambda_{0}$ and temperatures fairly agrees with the numerical calculations (using trial doublet states) when $\rho<0.5$ (figures 7 and 8). As an illustration, only some truncated results for the dimensionless parameters are presented in tables II and III. As an indicative corollary we also note that for $\theta$ close to zero an appreciable $\lambda_{0}$ - dependence of all the parameters is found, while the $f_{0}$ - dependence of these parameters (except for $J_{0}$ ) can be ignored (cl.[5]).

TABLE II

\begin{tabular}{cccccccc}
\multicolumn{2}{l}{$\lambda_{0}=0.1 ; f_{0}=0.1$} \\
\hline$\theta$ & $\Omega 10^{-3}$ & $J_{0}$ & $\eta$ & $y$ & $\sigma^{z}$ & $\sigma^{x}$ & $P_{s}$ \\
\hline 0.020 & 0.190 & 0.075 & 0.866 & 0.041 & 0.999 & 0.002 & 0.865 \\
0.040 & 0.220 & 0.074 & 0.858 & 0.045 & 0.939 & 0.003 & 0.805 \\
0.060 & 0.320 & 0.070 & 0.837 & 0.056 & 0.617 & 0.004 & 0.516 \\
0.080 & 0.530 & 0.064 & 0.803 & 0.072 & $0.1 \cdot 10^{-6}$ & 0.006 & $0.8 \cdot 10^{-7}$ \\
0.100 & 0.110 & 0.055 & 0.744 & 0.098 & $0.1 \cdot 10^{-6}$ & 0.011 & $0.8 \cdot 10^{-7}$
\end{tabular}


TABLE III

\begin{tabular}{cccccccc}
\multicolumn{2}{c}{$\theta=0.001 ; f_{0}=0.1$} \\
\hline$\lambda_{0}$ & $\Omega 10^{-3}$ & $J_{0}$ & $\eta$ & $y$ & $\sigma^{z}$ & $\sigma^{x}$ & $P_{s}$ \\
\hline 0.100 & $0.19 \cdot 10^{-3}$ & 0.075 & 0.866 & 0.041 & 1.000 & 0.002 & 0.866 \\
0.200 & $0.18 \cdot 10^{-1}$ & 0.043 & 0.653 & 0.095 & 0.903 & 0.430 & 0.590 \\
0.300 & $0.57 \cdot 10^{-1}$ & 0.037 & 0.609 & 0.122 & $0.1 \cdot 10^{-6}$ & 1.000 & $0.61 \cdot 10^{-7}$ \\
0.400 & 0.106 & 0.036 & 0.599 & 0.151 & $0.1 \cdot 10^{-6}$ & 1.000 & $0.60 \cdot 10^{-7}$ \\
0.500 & 0.164 & 0.037 & 0.606 & 0.177 & $0.1 \cdot 10^{-6}$ & 1.000 & $0.61 \cdot 10^{-7}$
\end{tabular}

\subsection{Remarks on the applicability of the model}

The model dealt with essentially represents a generalization of the De Gennes model (cf. [5-7]). Here, both the dependence of the model double-well potential on temperature $T$ and the quantum properties of the system $\left(\lambda_{0}^{2} \sim \hbar^{2} / m\right)$ have been considered. The principal approximation consists in the fact that the total spectrum is divided into two parts: a low-lying part of a doubly split level due to tunnelling and the other part of higher excited states. The mutual influence of these two parts of the spectrum is, then, taken into account in the frames of mean-field type approximations. The parameters of the pseudospin Hamiltonian (4.6), $\Omega$ and $J_{i i^{\prime}}$, depend essentially on the state of the phonon subsystem (the populations of the higher levels) through the effective one-particle potential (4.17). And vice versa, the state of the phonon subsystem depends on the state of the pseudospin subsystem, since the phonon frequency (4.20) depends on the mean square displacement of the equilibrium positions of vibrations $\left\langle r_{i}^{2}\right\rangle_{0}$ (4.19), depending otherwise on $\sigma^{x}$.

For describing a phase transition in the system it is necessary to solve a set of equations (4.15)-(4.21). A numerical solution was obtained in Ref. [5]. At $\lambda_{0}=0.1$ there exists a double-minimum potential with two closely situated levels below the potential barrier. Degeneracy of the ground state is removed by a quantum tunnelling of the particle (cell). When $\lambda_{0}=0.3$, the kinetic energy of the particle (or cell) is so high that the energy levels are situated above the potential barrier. In this case, evidently, the representation of the effective displacement in the form (4.1) loses sense. Moreover, since the energy spectrum of a particle in a local (double-well) potential has quite a complex structure [5,20], such a separation has merely an interposal character, i.e. being physically inapplicable for the temperature region, when $\hbar \Omega \sim \hbar \omega_{0}$. In particular, one could expect a more complex renormalization of the pseudospin parameters in real order-disorder compounds, especially when the excited atomic states lie in the critical temperature region, $k_{\mathrm{B}} T_{c} \sim J_{0} \sim \hbar \omega_{0}$.

Summing up the theoretical and numerical analyses presented, it should be pointed out that the generalised model satisfactorily reveals the essential features of both the order-disorder and the displacive types of SPTs at finite and zero temperatures. The true quantum-mechanical situation, i.e. the tunnelling (local or effective) motion of active atoms (or cells), is treated properly with respect to the approximations applied and the limiting cases considered. In particular, 
the numerical results show [5] that in the approximation of strong anharmonicity $\left(f_{0} \ll 1\right)$, as in the case of the displacive limit (see Subsection 3.5), zero vibrations can violate the ordered state, thus, not arising even at $T=0$. In other words, quantum fluctuations may suppress the phase transition in the system, as one can see from figure 9 in which the dependence of the "total" order parameter $P_{s}(4.38)$ on $\lambda_{0}$ is shown for two values of the coupling constant $f_{0}$.

Concluding this section, we believe that the present model, as analysed by means of a more accurate selfconsistent procedure, in addition to its extension in the spirit of the central

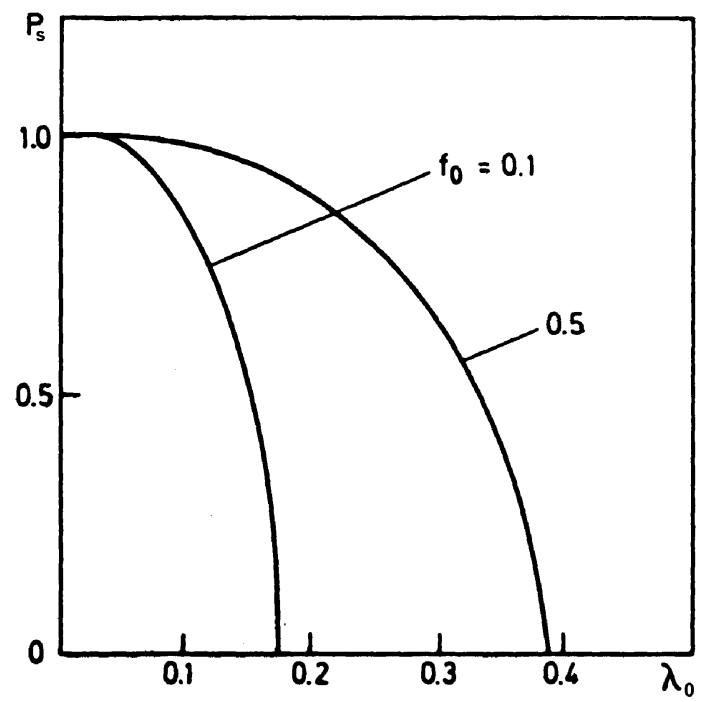

Figure 9. The dependence of $P_{s}$ on the quantum parameter $\lambda_{0}$ for $T=0$. peak dynamics and solitary waves, can complete the analytic description of the critical dynamics and yield a deeper insight into the nature of SPTs in general.

\section{Nonlinear effects and the incomplete softening of critical modes}

\subsection{A need for domain-wall soft mode dynamics}

The principal role of nonlinear effects at STPs was originally pointed out in [21]. Thus, the exact solution for a one-dimensional $(d=1)$ lattice shows that besides phonons (the softening of which causes a displacive phase transition) there are also nonlinear (soliton-like) excitations which independently of (or additionally to) phonons can lead to the instability of the lattice. This fundamental inference is also consistent with molecular dynamics simulations (from $d=1$ to $d=4$ ) [22], as well as with the results of the exact dynamics of a few coupled quartic oscillators [23]. In a series of subsequent theoretical works (cf. $[7,11]$ ) it was envisaged that with the onset of criticality a crossover from a "displacive" to an "order-disorder" regime could occur manifested in the formation of long-lived clusters of the precursor order. The existence and dynamics of such clusters give rise to the central peak (i.e. the nonclassical critical behaviour) and to the fact that the soft mode is suppressed due to the local symmetry violation (see, e.g., [24]). While such a physical picture is conceptually quite acceptable, it is still not analytically substantiated and also lacks an indisputable experimental verification. Nevertheless, the authors [24-26] interpreting EPR-experiments on monondomain-transforming $\mathrm{SrTiO}_{3}$ (through motional narrowing, using $\mathrm{Fe}^{3+}-\mathrm{V}_{0}$ centres) drew attention to the challenging accord between theoretical and experimental estimates of a non-vanishing soft- 
mode frequency at the phase transition temperature $\left(T_{c}\right)$, thus, prompting further progress in comprehensive SPT investigations. Apparently, such a coincidence corroborates the model description [1-5,21-26] by which the local normal coordinate $s_{i}(\mathrm{t})$ is decomposed into a slow momentary quasirest position $\left(r_{i}(\mathrm{t})\right)$ and a comparatively fast deviation $u_{i}(\mathrm{t})$ (around $r_{i}(\mathrm{t})$ ) of the phonon (quasiharmonic) type. In the frames of such ideas, our preceding works $[1-5,7]$ were devoted to analytical studies of the SPT description within a single universal model, using in addition the Bogolyubov variational approach. Thus, a novel interpolar scheme attempting to include all the intriguing features of the SPT, i.e. statistical order-disorder, tunnelling (real and effective) and phonon excitations has been proposed (see Section 3 and 4). To describe a possible order-disorder behaviour of the traditionally displacive SPT (revealed in an incomplete phonon softening) the adjustment of the previous models is accomplished by employing both the solitary-waves and renormalization-group (RG) techniques (cf. [5-7]). Note that the RG-method has been used in this sense only for order-disorder systems [27].

\subsection{The critical region}

The unified theory of SPTs afforded by the model studies which were surveyed in the preceding sections can be qualified as applicable within the same limits as the Landau theory. Such a description provides a qualitative picture of SPTs, particularly of traditionally limiting types, as well as for some model parameters of mixed transitions which do not belong to either of two extreme cases. However, like the previous theories, it does not permit investigation of their properties in the critical region (cf. also $[7,11]$ ).

Investigation of the phase transition (for instance, in strontium titanate $\mathrm{SrTiO}_{3}$, with the aid of inelastic neutron scattering (cf. [7])) reveals that the excitation spectrum in the critical region is more complex than that which follows from the classical theory. The scattering intensity exhibits, besides two side peaks corresponding to the soft mode, a narrow peak at zeroth energy transfer. As $T \rightarrow T_{c}$ the intensity of this central peak increases sharply, while its width decreases. The frequency of the soft mode decreases up to a certain finite value at $T=T_{c}$. Subsequent experiments demonstrate that this pattern is characteristic of all SPTs.

At present the nature of the central peak still remains to be clarified. The reasons for its appearance are divided into two sorts: intrinsic (fluctuations of the phonon density, nonlinear effects, etc.) and extrinsic (defects, impurities, surface). The results of numerous studies of this question, as well as an extensive bibliography, can be found in the reviews (cf. $[7,11]$ ). It can be concluded from the results of these works that a system in the critical region is characterized by two time scales, instead of one, as in the conventional theory of the soft mode. In addition to the relatively short (noncritical) relaxation time of the system characterizing the soft phonon, there exists a large (critical) relaxation time characterizing the narrow central peak. In this section, on the basis of model (2.10), the rise of a double-time evolution at a displacive or an order-disorder phase transition in a 
perfect (defectless) crystal is considered.

In accordance with the universality hypothesis, model (2.10) belongs to the Ising universal class, i.e. it shows the same critical behaviour as the Ising model. This means that for $f_{0} \gg 1$, at least in the critical region, when $T>T_{c}$, a shortrange order should arise in the system leading to a non-Gaussian distribution of the order parameter. Such a result already follows from the first applications of the RG-method to model [11]. Utilization of this method for calculating the coordinate distribution function for blocks shows [28] that in a two-dimensional case $(d=2)$ it has a pronounced structure with two maximums. When $d=3$, the distribution has a single maximum but of a non-Gaussian type.

From calculations [28] it follows that a phase transition in model (2.10) in the limit $f_{0} \gg 1$ exhibits clearly pronounced features of an order-disorder transition at $d=2$ and close to the features of such a transition at $d=3$. A detailed investigation of the transition from the displacive regime to the order-disorder regime in model (2.10) for $d=1$ and $d=2$ was carried out in [29] by applying the RG-method in the direct space. It was shown that on the phase diagram $\left(0 \leqslant T \leqslant \infty, 0 \leqslant f_{0} \leqslant \infty\right)$ there exists a crossover line $T_{0}\left(f_{0}\right)$ with the finite points $T=0$ and $f_{0} \sim 0.5$. Below this line the system goes over from the displacive regime to the Ising one in which the depth of potential minima increases after each iteration of the RGtransformation. In this region of parameters $T$ and $f_{0}$ the system has a fixed Ising point. When $d=2$, it is also established that, at least for $d \leqslant 2$, the system described by equation (2.10) undergoes, in the displacive limit $\left(f_{0} \gg 1\right)$ and in the temperature region $T_{c}<T<T_{0}$, a transition to the order-disorder regime exhibiting the Ising critical behaviour. However, the RG-method does not permit a description of the excitation spectrum in this region. For this purpose other methods should be applied (see subsection 5.3).

Investigation of model (2.10) by analytical methods [21] for $d=1$ and by the method of molecular dynamics [22] (cf. also [6,7,11]) for $d \leq 4$ reveals that a transition to the order-disorder regime is accompanied by the appearance of short-range order clusters, or microdomains, with different polarizations of the order parameter. A natural conjecture was to assume that such clusters appear at $T=T_{0}\left(f_{0}\right)[29]$. When $T>T_{0}\left(f_{0}\right)$, the system is characterized by small vibrations relative to the position of the hump in the effective one-particle potential. As the temperature drops lower than $T_{0}$, clusters walls are formed which put bounds for the regions of dimensions of the correlation length. At $T=T_{c}\left(f_{0}\right)$ the system undergoes a continuous phase transition to an ordered state with Ising critical exponents. When $f_{0} \leqslant 0.5$, the excitations most significant for the system are the ones (soliton waves-like) which appear due to the formation of walls. The influence of phonons on this process is of no principal importance, since the potential minima are so deep that jumps (i.e. tunnelling "crossings") from one well to another are extremely rare. The system is Ising-like at all temperatures. When $f_{0} \gg 1$, the system is Ising-like within the region $T_{c}<T<T_{0}$. In this temperature region the principal excitations are the ones related to the formation of walls between clusters with different polarizations of the order parameter. At $T>T_{0}$ the walls between 
the clusters start vanishing, and phonon excitations become the most significant ones.

Now one can envisage a qualitative picture of the behaviour of the excitation spectrum. When $T \geqslant T_{0}$ (the displacive regime), the particles vibrate relatively to their symmetric equilibrium positions; the spectrum exhibits two peaks (phonon emission and absorption in scattering intensities) corresponding to the frequency of soft phonons and determined by the poles of the standard Green function, $D_{q}(\omega)=\left[\omega^{2}-\omega_{q}^{2}-M_{q}(\omega)\right]^{-1}$ (unlike, e.g., equation (3.27), self-energy operator $M_{q}(\omega)$ is introduced herein). At $T_{c}<T \leqslant T_{0}$ the system passes to the orderdisorder regime in which the particles vibrate around quasiequilibrium positions displaced relatively to the lattice sites in the symmetric phase: there arise clusters of different polarizations serving as the initial nuclei of the new phase; under the stabilizing influence of the clusters the high-temperature phonon evolves into its low-temperature counterpart without exhibiting a complete softening. The critical slowing down of the system is provided by the relaxation motion of the cluster walls contributing to the energy (scattering) spectrum and the central quasielastic component $[7,11]$.

\subsection{Domain walls and phonons in an inhomogeneous medium}

Now let us consider in greater detail the range of temperatures $T_{c}<T<T_{0}$ within which the system becomes inhomogeneous with respect to the distribution of equilibrium positions $r_{i}$. These equilibrium positions have for each cell their particular value and are practically quasiequilibriums, meaning that they can change with time.

We consider the widely used model Hamiltonian ((2.10), i.e. (4.14)):

$$
H=\sum_{i}\left(\frac{P_{i}^{2}}{2 m}-\frac{A}{2} s_{i}^{2}+\frac{B}{4} s_{i}^{4}\right)+\sum_{i j} \frac{1}{4} \varphi_{i j}\left(s_{i}-s_{j}\right)^{2},
$$

with the local normal coordinate $s_{i}$ represented in the form $((3.1)$, i.e. $(4.1))$ :

$$
s_{i}=r_{i}+u_{i}
$$

$r_{i}$ is now defined by

$$
r_{i}=\left\langle s_{i}\right\rangle_{p h}=\frac{1}{Z_{p h}} \operatorname{Sp}_{\left\{u_{i}\right\}}\left(e^{-H / T} s_{i}\right)
$$

where symbol $\langle\ldots\rangle_{p h}$ (i.e. indication "Sp$\left\{u_{i}\right\}$ ") stands for the quantum-statistical averaging with respect to quasiharmonic deviations $u_{i}$ (around quasiequilibrium positions $r_{i}$ ). Note that representation (5.2) is based on the fact that variables $r_{i}(t)$ and $u_{i}(t)$ have distinct time scales: the large (i.e. "slow") $r_{i}(t)$ and small (i.e. "fast") $u_{i}(t)$ displacements correspond to the quasi-soliton (domain-wall) and phonon type excitations, respectively. 
For further consideration, similarly to the ansatz in subsection 4.2 , it is convenient to represent the Hamiltonian (5.1) as a sum of two Hamiltonians describing the configurational (index $c$ ) and phonon (index $p h$ ) parts:

$$
\begin{gathered}
H_{0}=H_{c}+H_{p h}, \\
H_{c}=\sum_{i}\left[\frac{p_{i}^{2}}{2 m}-a_{i} \frac{A}{2} r_{i}^{2}+\frac{B}{4} r_{i}^{4}\right]+\frac{1}{4} \sum_{i j} \varphi_{i j}\left(r_{i}-r_{j}\right)^{2}, \\
H_{p h}=\sum_{i}\left[\frac{-\hbar^{2}}{2 m} \frac{\partial^{2}}{\partial u_{i}^{2}}-\Delta_{i} \frac{A}{2} u_{i}^{2}\right]+\frac{1}{4} \sum \varphi_{i j}\left(u_{i}-u_{j}\right)^{2} .
\end{gathered}
$$

The variational parameters $a_{i}$ and $\Delta_{i}$ which take into account the coupling of two subsystems are determined (in the spirit of the Bogolyubov variational approach):

$$
a_{i}=1-3(B / A)\left\langle u_{i}^{2}\right\rangle_{0}, \quad \Delta_{i}=3(B / A)\left\langle r_{i}^{2}\right\rangle_{0}-a_{i} .
$$

By the same variational approach, or directly from the equilibrium condition (for the system described by the initial model Hamiltonian (5.1)),

$$
\left\langle i \frac{d}{d t} P_{i}(t)\right\rangle_{p h}=\left\langle\left[P_{i}, H\right]\right\rangle=0,
$$

equivalent to minimization of the free energy for a given configuration $\left\{r_{i}\right\}$,

$$
\frac{\partial}{\partial r_{i}} F\left(\left\{r_{i}\right\}\right)=0 ; F\left(\left\{r_{i}\right\}\right)=-k_{\mathrm{B}} T \ln \operatorname{Sp}_{\left\{u_{i}\right\}} e^{-H / T}
$$

one obtains the difference equation (compare with equation (3.12)):

$$
\eta_{i}\left[\eta_{i}^{2}-a_{i}(T)\right]+\sum_{j} f_{i j}\left(\eta_{i}-\eta_{j}\right)=0 ; f_{i j}=\varphi_{i j} / A
$$

by which the reduced quasiequilibrium positions (i.e. the local order parameters) $\eta_{i}=(B / A) r_{i}$ are determined as site $(i)$-dependent functions of temperature. Thus, besides the zero solution $\eta_{i}=0$, equation (5.10) can have a solution varying in space if the boundary conditions are chosen appropriately.

To introduce phonons into an inhomogeneous system, it is necessary to perform the structural averaging over all the configurations $\left\{r_{i}\right\}$. We shall restrict ourselves to the virtual (average)-crystal approximation in which the random one-particle potential is substituted by the average perturbation potential in the effective lattice invariant under translations:

$$
\left.\bar{\Delta}=3(B / A) \overline{\left\langle\eta_{i}^{2}\right.}\right\rangle-\bar{a}, \quad \bar{a}=1-3(B / A) \overline{\left\langle u_{i}^{2}\right\rangle},
$$

where the bar denotes the configurational average and index "0" being omitted here and further; the correlation function $\left\langle\overline{u^{2}}\right\rangle$ of displacements of the effective ("average") lattice with the vibration frequency

$$
m \bar{\omega}_{q}^{2}=A\left(\bar{\Delta}+f_{0}-f_{q}\right) ; f_{q}=\sum_{j} f_{i j} e^{\underline{i q\left(l_{i}-l_{j}\right)}}
$$


in accordance with equation (4.18) is given by

$$
\overline{\left\langle u^{2}\right\rangle}=\frac{1}{m N} \sum_{q} \frac{1}{2 \bar{\omega}_{q}} \operatorname{coth} \frac{\bar{\omega}_{q}}{2 T}
$$

Thus, the behaviour of phonons in the region $T_{c}<T \lesssim T_{0}$ is determined by a self-consistent set of equations (5.10)-(5.13).

We shall consider a quasi-one-dimensional case when the reduced displacement $\eta_{i}$ depends only on the coordinate along certain chains, but the phonons in this case are three-dimensional. Such a model is quite specific in the sense that, as it will be demonstrated below, no ordered phase exists in it at finite temperatures. However, $T=0$ can be considered as the critical temperature, while taking the temperature range $T>0$ to be a symmetric-phase region.

\section{a) The case of a weak coupling limit*}

In a weak-coupling limit $\left(f_{0} \ll 1\right)$, a transition of the order-disorder type at critical temperature $T_{c}$ can be described by the effective Ising model [12] via pseudospin variables: $\eta_{i}=\sigma_{i} \sqrt{a}, \sigma_{i}= \pm 1$. As the role of fluctuations is not significant in this case [5], i.e. $\left\langle u^{2}>/(A / B) \sim T /\left(A^{2} / B\right) \ll 1\right.$ at $T \sim T_{c} \sim f_{0}\left(A^{2} / B\right) \ll$ $A^{2} / B$, one estimates $a \approx 1$, and, consequently, $T_{c} \ll T_{c}^{0}, T_{c}^{0}$ to be the critical temperature of a displacive SPT at which $a\left(T_{c}^{0}\right) \equiv 0$ (compare $T_{c}$ and $T_{c}^{0}$ with equations (4.24) and (4.26), respectively). Furthermore, when the ground-statequantum splitting within a local double-well potential plays a predominant role in the dynamics at low temperatures (e.g., when $T \sim T_{c} \approx f_{0} A^{2} / B \ll U / k_{\mathrm{B}}$ ), then the Ising model with a transverse field (IMTF) is more adequate, accommodating itself the additional "intra-well" degree of freedom [6].

\section{b) The case of a strong coupling limit}

In a strong coupling limit $\left(f_{0} \gg 1\right)$ (we are mainly interested in throughout this subsection), a slow varying $\eta_{i}$ evolves, e.g., $\left|\eta_{i}-\eta_{j}\right| \sim 1 / f_{0} \ll 1$ (cf. equation (5.10)), so a the version $\eta_{i} \rightarrow \eta(x)$ in equation (5.10), is possible:

$$
\eta(x)\left[\eta^{2}(x)-a(x, T)\right]+\sum_{\alpha \beta} c_{\alpha \beta} \partial^{2} \eta(x) / \partial x_{\alpha} \partial x_{\beta}=0,
$$

where elasticity moduli are introduced

$$
c_{\alpha \beta}=\sum_{j} f_{i j} \frac{1}{2}\left(x_{i}^{\alpha}-x_{j}^{\alpha}\right)\left(x_{i}^{\beta}-x_{j}^{\beta}\right) .
$$

In a pseudo one-dimensional case $(d=1)$ (the only tractable analytically), the above equation (5.14) becomes

$$
c \frac{\mathrm{d}^{2} \eta(x)}{\mathrm{d} x^{2}}+\eta^{3}(x)-a(x, T) \eta(x)=0,
$$

\footnotetext{
${ }^{*}$ Henceforth, for convenience, the designations by bars are omitted
} 
where in the mean-field approximation $a(x, T)$ is determined by

$$
\begin{aligned}
a(x) & =1-3 y(x) \\
y(x, T) & =(B / A)\left\langle u^{2}\right\rangle=\left(\lambda / 2 \sqrt{\Delta(x)+f_{0}}\right) \operatorname{coth}\left(\lambda \sqrt{\Delta(x)+f_{0}} / 2 \tau\right), \\
\Delta(x) & =3\left[\eta^{2}(x)+y(x)\right]-1=3 \eta^{2}(x)-a(x),
\end{aligned}
$$

where $\lambda=\hbar \sqrt{A / m} /\left(A^{2} / B\right)$ is a quantum parameter (equation (2.16)) and $\tau=$ $T /\left(A^{2} / B\right)$ is a reduced temperature.

The system of equations $(5.16),(5.17)$ differs from the corresponding ones in [30] as thermal (in addition to quantum) effects are taken into account herein. Hence, assuming homogeneous boundary equilibrium positions, $\eta_{0}=\eta(x \rightarrow \pm \infty)$, one obtains a soliton solution:

$$
\eta(x) \approx \pm \sqrt{a(T)} \tanh \frac{x}{\left(\sqrt{2} \xi_{0}\right) / \sqrt{a(T)}}, \quad \xi_{0}=\sqrt{c}=c_{0} \sqrt{m / A l^{2}},
$$

where $c$ is the elasticity modulus and $c_{0}=l \sqrt{\varphi_{0} / 2 m}$ is the maximum soliton velocity (the velocity of sound in the system); $\eta(x)$ describes a fixed wall between clusters, e.g. the transition region between stable particle (cell) positions $r_{0}=$ $\pm \sqrt{a A / B}$.

It is easily seen that phonon fluctuations renormalise (through $a(T)$ ) the soliton amplitude, $\eta_{0}=\sqrt{a(T)}<1$, and its width, $\sqrt{c / a(T)}>\xi_{0}$. The variation within a domain wall $\left(x<\xi_{0}\right)$ can be easily (iteratively) accounted for [30] but the form of the solution (5.18) is not substantially altered. From the demand for the homogeneous solution $\eta(x)=\eta$ (being the order parameter actually) to vanish identically and using equation (5.16) one estimates the phase transition temperature (i.e. of the soft-mode condensation, $T_{c}^{0} \sim\left(f_{0} / 3\right) A^{2} / B$, since $\left.y \sim T B / f_{0} A^{2}\right)$.

The inhomogeneous solution (5.18) also admits another type of a phase transition corresponding to instability of the whole system with respect to differently oriented clusters $(\eta(x) \sim \pm \sqrt{a})$. In that case the displacive SPT can be viewed as a transition of the order-disorder type, i.e. at $T=T_{c}<T_{c}^{0}: \eta=\langle\eta(x)\rangle_{c}=0$, but $\left\langle\eta^{2}(x)\right\rangle_{c} \approx a\left(T_{c}\right) \neq 0$, symbol $\langle\ldots\rangle_{c}$ denoting the average over all the clusters. This picture is dictated by non-linearity of the model. In addition, it is consistent with the results of the molecular dynamics methods from $d=1$ to $d=4$ dimensions (cf. $[6,7,11])$. To corroborate it more profoundly, in the next section we will consider the behaviour of the probability distribution function of quasiequilibrium positions $\mathrm{P}(\eta)$ in the limit $T \rightarrow T_{c}$.

\subsection{The (quasi)soliton correlations}

The time-dependent solution for $\eta(y)$ is obtained from (5.18) with the aid of the Lorenz transformation, i.e. by the substitution $y \rightarrow(x-v t) / \sqrt{1-v^{2} / c_{0}^{2}}$, and corresponds to quasisoliton propagating along a chain with the velocity $0 \leqslant v \leqslant$ $c_{0}$. In the non-relativistic limit $\left(v \ll c_{0}\right)$ the total energy $E$ of such a soliton is composed [21] (see also [7]) of the potential $\left(E_{0}\right)$ and the kinetic $\left(E_{k}=m^{*} v^{2} / 2\right)$ energies, respectively: 


$$
E=E_{0}+\frac{m^{*} v^{2}}{2}, \quad E_{0}=\frac{A^{2}}{B} \frac{2 \sqrt{2} \xi_{0}}{3 l} a^{3 / 2} ; \quad m^{*}=\frac{2 \sqrt{2}}{3} m \frac{A / B}{l \xi_{0}} a^{3 / 2},
$$

where $m^{*}$ is the effective mass of the quasisoliton (the cluster wall) and $l$ - the lattice constant.

When $\left|x_{0 i}-x_{0, i+1}\right| \gg \xi_{0}, x_{0 i}$ is the domain-wall centre, i.e. when the density of the cluster walls is low, equation (5.16) has the approximate partial solution:

$$
\eta_{N_{c}}\left(x, t ;\left\{x_{0 i}\right\},\left\{v_{i}\right\}\right)=\sqrt{a} \prod_{i=1}^{N_{c}} \tanh \frac{x-x_{0 i} \mp v_{i} t}{\sqrt{2} \xi / \sqrt{a}},
$$

where $\xi=\xi_{0} \sqrt{1-v^{2} / c_{0}^{2}}$. Solution (5.20) describes a configuration consisting of $N_{c}$ clusters.

In the low temperature region $T \ll E_{0}$ the partition function of the system of cluster walls (5.20) assumed as a "gas consisting of quasiparticles (quasisolitons)" is given by (cf. [7]):

$$
Z_{c}=\exp \left[L\left(\frac{2 \pi T}{m^{*} D^{2}}\right)^{1 / 2} e^{\left(\mu-E_{0}\right) / T}\right],
$$

where $D$ is a normalizing factor in the phase space of the positions of cluster walls $x_{0 i}$ and their velocities $v_{i}$.

By making use of the fact that the chemical potential of a quasisoliton gas $\mu=0$ and of expression (5.21), the density of the cluster walls is of the form:

$$
\overline{n_{c}}=\frac{\overline{N_{c}}}{L}=\frac{T}{L} \frac{\partial}{\partial \mu} \ln Z_{c}=\left(\frac{2 \pi T}{m^{*} D^{2}}\right)^{1 / 2} e^{-E_{0} / T} .
$$

Now, let us consider the configurational averaging of quasiequilibrium positions, after [31]. As to the initial condition at $T=0$, it is assumed that there exists one domain with $\eta(x, 0)=\eta_{0}=\sqrt{a}$. At temperatures differing from zero, clusters with quasiequilibrium positions of different polarizations begin to appear in the system, so one finds:

$$
\langle\eta(x, 0)\rangle_{c}=Z_{c}^{-1} \sqrt{a}=\left\{\begin{aligned}
\sqrt{a}, & T=0 \\
0, & T>0 .
\end{aligned}\right.
$$

Thus, the average value (thermodynamic and configurational) of the equilibrium position of a particle in a one-dimensional model (5.1) is equal to zero at any nonzero temperature.

The average squared values of the equilibrium positions are calculated likewise:

$$
\left\langle\eta^{2}(x, 0)\right\rangle_{c}=a e^{-2 \bar{n}_{c} \xi_{0} \sqrt{2} / \sqrt{a}} .
$$

The adopted procedure for configurational averaging also permits one to obtain the dynamic correlation function as follows (cf. [7,31]) : 


$$
\langle\eta(x, t) \eta(0,0)\rangle_{c}=a \exp \left[-2 e^{\frac{E_{0}}{T}} \int_{-\infty}^{\infty} \frac{\mathrm{d} v}{D} e^{-\frac{m^{*} v^{2}}{2 T}}(x \mp v t) \operatorname{coth} \frac{x \mp v t}{\sqrt{2} \xi_{0} / \sqrt{\bar{a}}}\right] .
$$

The static correlation function that follows from (5.25) at $t=0$ upon integration over the velocities acquires the form:

$$
\langle\eta(x, 0) \eta(0,0)\rangle_{c}=a \exp \left[-\bar{n}_{c} 2 x \operatorname{coth} \frac{x}{\sqrt{2} \xi_{0} / \sqrt{a}}\right] .
$$

When $x \gg \xi_{0} / \sqrt{a}$, expression (5.26) can be presented in the form obtained by the transfer-matrix method [21],

$$
\langle\eta(x, 0) \eta(0,0)\rangle_{c}=a e^{-x / \xi_{c}},
$$

where $\xi_{c}=1 / 2 \bar{n}_{c}$ is nothing but the correlation length in the system (as $\left.T \rightarrow T_{c}=0, \xi_{c} \rightarrow \infty\right)$.

\subsection{The soft mode and the central peak}

In the preceding sections lattice vibrations were mainly considered in the classical (high-temperature) limit. At low temperatures (or small effective mass) it is necessary to take into account zero-point (quantum) vibrations. The quantum properties of the model are characterized by the parameter $\lambda \sim \hbar / \sqrt{m}$ (see equations $(2.16),(4.30))$, which plays the same role as temperature: when the coupling constant $f_{0}$ is fixed, the system may become unstable with respect to the propagation of both phonons $\left(\bar{\omega}_{q s}^{2}<0\right)$ and quasisolitons $(E<0)$, if $\lambda>\lambda^{c}$, even if $T=0$. The effect consisting in the suppression of a phase transition by quantum fluctuations is well-known. For example, the absence of a ferroelectric transition in $\mathrm{SrTiO}_{3}$ at normal pressures is most likely due to the above said effect.

Let us find the critical parameters determining the limit imposed on the dynamic stability of the system, which requires the phonon energy (5.12) and the soliton energy (5.19) to turn to zero. We introduce the dimensionless variables:

$$
\bar{\Omega}_{q}^{2}=\frac{\bar{\omega}_{q}^{2}}{A / m} ; \varepsilon_{0}=\frac{E_{0}}{A^{2} / B} ; \theta=\frac{T}{A^{2} / B} ; \lambda=\frac{\hbar \sqrt{A / m}}{A^{2} / B}
$$

and rewrite equation (5.13) in the form:

$$
\frac{B}{A}\left\langle u^{2}\right\rangle=\frac{\lambda}{2} \int_{0}^{\omega_{m}^{2}} \frac{g\left(\omega^{2}\right) \mathrm{d} \omega^{2}}{\sqrt{\Delta+\omega^{2}}} \operatorname{coth} \frac{\lambda \sqrt{\Delta+\omega^{2}}}{2 \theta},
$$

where the phonon frequency density

$$
g\left(\omega^{2}\right)=(1 / N) \sum_{q} \delta\left(f_{0}-f_{q}-\omega^{2}\right)
$$

is introduced for $\omega^{2} \leqslant \omega_{m}^{2}=2 f_{0}(A / m)$. 
Consider a phase transition in the centre of the Brillouin zone at $q=0$. Then, in the symmetric phase, in the absence of clusters (when $\left\langle\eta^{2}\right\rangle_{c}=0$ ) the condition for determining the critical parameters has the form $3 B\left\langle u^{2} r a / A=1\right.$, from which for the Debye spectrum $g\left(\omega^{2}\right)=3 \omega / \omega_{m}^{3}$ one obtains:

$$
\lambda_{0}(T=0) \approx \sqrt{f_{0}} ; \theta_{0}=\frac{T_{0}(\lambda \rightarrow 0)}{A^{2} / B} \approx \frac{f_{0}}{9} ; \varepsilon_{0}(\lambda \rightarrow 0, T \rightarrow 0) \approx \sqrt{f_{0}}
$$

Thus, application of the presented approach is limited by the following values of the model parameters $\lambda_{0}$ and $f_{0}$ and the reduced temperature:

$$
f_{0} \gg 1 ; \lambda_{0} \ll \sqrt{f_{0}} ; 0.05 \sqrt{f_{0}}<\theta \ll \sqrt{f_{0}} .
$$

The temperature behaviour of the phonon energy is determined by a selfconsistent set of equations (5.11)-(5.13), (5.19), (5.22), (5.24), and (5.26). The numerical solution of this set of equations for the model parameters $f_{0}=10$ and $\lambda_{0}=0.1[7,31]$ shows that for $\theta>\theta_{0} \approx 0.5$, the soft mode behaves as in the classical theory. When $\theta \approx \theta_{0}$, the "high-temperature" phonons (phonons in the symmetric phase) become unstable, which is related to the appearance of clusters (quasisolitons) in the system. In the presence of clusters the phonons behave like "low temperature" phonons (phonons in the nonsymmetric phase). As $\theta \rightarrow \theta_{c}=0$, instead of decreasing as in the classical theory, the soft mode frequency increases and tends to a finite value. A qualitative result provides the observed incomplete softening of the soft(critical) mode as a consequence of inhomogeneity of the system which leads to a nonzero value of the averaged square equilibrium positions $\left(\left\langle\eta^{2}\right\rangle_{c} \neq 0\right)$. At the same time the order parameter (for example, spontaneous polarization in the case of ferroelectrics) that is proportional to the average displacement $\langle\eta\rangle_{c}$ remains equal to zero till $\theta_{c}=0$.

Now let us consider the behaviour of the central peak as an intrinsic feature of the critical (SPT) dynamics apart from the revealed in the spectral density function. In various scattering experiments the scattering function $S(q, \omega)$ is measured as one being expressed through the dynamic correlation function. In the case of quasisolitons it is represented as the double (in $q$ and $\omega$ ) Fourier transformation of the correlation function (5.25). Within the framework of the variational approach and approximations applied, the scattering function of the SPT-system involves three singled peaks-two sharp side peaks corresponding to phonon energies (5.12) and a central one at $\omega=0$, due to the dynamics of the cluster walls (quasisolitons). As to the quasisolitons (assumed as a "gas of quasiparticles"), the two limit regimes of their behaviour are feasible: collisionless and diffusive. We shall not comment on the collisionless regime (otherwise corresponding to a free motion of quasisolitons) as it is of a less physical interest. Diffusive behaviour itself corresponds to the asymptotic $t \rightarrow \infty$ in the Fourier $(q, \omega)$ - transformed correlation function (5.25). Assuming the correlation function (5.25) to be independent of the coordinate within the limits of the correlation length $\xi_{c}$ and integrating (in $x$ and 
t) its Fourier $(q \omega)$ component, the scattering function in the critical region (index $c r)$ is estimated as follows $[7,31]$ :

$$
S_{c r}(q, \omega) \approx a \frac{A}{B} \frac{\sigma_{\lambda}(q)}{\pi} \frac{\gamma}{\omega^{2}+\gamma^{2}}, \quad \gamma=\frac{4 T}{m^{*} D} e^{-E_{0} / T},
$$

where $\sigma_{\lambda}(q)$ is an approximate space transformation of the correlation function within the correlation-length limits. Thus, in the case of a simple diffusion of the cluster walls there should be observed, in the scattering function, a central peak of the Lorentzian form and half-width $\Gamma_{0} \approx \gamma\left(\Gamma_{0} \approx \bar{v} / \zeta\right.$, where $\bar{v}$ is the mean velocity of the walls and $\zeta$ is the mean distance between them). As the temperature decreases, the height of the central peak increases, while its half-width decreases. In the limit, as $T \rightarrow 0, S_{c r}(q, \omega)$ (5.33) transforms into a $\delta$-function.

Being analytically intractable, the proper scattering function for (quasi)solitons (index c) $S_{c}(q, \omega)$ versus temperature is obtained by a numerical integration (cf. $[7])$. For the chosen parameters of the model $\left(f_{0}=10, \lambda=0.1\right)$ and $q \neq 0(q / l=$ 0.016 ), the intensity of the central peak first increases as $T$ decreases (starting from $T_{0}$ ) and then starts decreasing at $T \rightarrow 0$. At $q=0$ the central peak has the same form as at finite $q$, but its intensity increases as $T \rightarrow T_{c}$, while its half-width $\left(\Gamma_{0}\right)$ decreases. Therefore, it follows that for finite $q$, in an ideal (defectless) lattice (in the symmetric phase, $T>0$ ), the contribution of moving cluster walls to the central peak decreases as $T \rightarrow T_{c}=0$. But the peak becomes a central (intensive) one when $q \rightarrow 0$. Thus, the analytical investigation of the one-dimensional model dynamics presented in this subsection confirms the qualitative picture and gives details of the phase transition which follow from the analysis based on the renormalization group method, as described above. Below we outline the application of the RGmethod to explain, rather analytically and in part quantitatively, the pronounced influence of cluster excitations on phonons and pseudomagnons, i.e. their impact on critical (SPT) dynamics in all.

\subsection{RG - predictions and domain-wall relaxation}

As it is evident from equation (5.10), the probability distribution function of quasiequilibrium positions, $P(\eta)$, is described by the configuration-potential energy of the system (see equation (5.1)). In the RG-analysis (index $r g$ ) it is convenient to use the dimensionless quantities $(r=a / c, g=1 / 4 c)$, so in the continuum version

$$
P_{r g}(\eta)=Z^{-1} \exp \left[-H_{r g}(\eta)\right]
$$

where

$$
H_{r g}(\eta)=\beta U_{r g}(\eta)=\frac{1}{2} \int \mathrm{d}^{d} x\left\{r \eta^{2}(x)+(\nabla \eta(x))^{2}+2 g \eta^{4}(x)\right\}, \beta=c / k_{\mathrm{B}} T,
$$

is the standard Landau-Wilson Hamiltonian.

As it is well known, at the critical point $\left(T_{c}\right)$ the stochastic field $\{\eta(x)\}$ (and correspondingly its distribution $P(\eta)$ ) is scaling-invariant, thus describing a distribution of large-size clusters. It is defined by the fixed point $\left(r^{*}, g^{*}\right)$ of the 
RG-transformation (R),

$$
R P_{r^{*} g^{*}}(\eta)=P_{r^{*} g^{*}}(\eta),
$$

wherefrom the first order in $\epsilon \equiv(4-d)$ expansion yields (cf. [6] and Refs. therein)

$$
r^{*}=-\frac{n+2}{n+8} \varepsilon+O\left(\varepsilon^{2}\right) ; \quad g^{*}=\frac{8 \pi^{2}}{n+8} \varepsilon+O\left(\varepsilon^{2}\right) ; \quad n=d,
$$

where $n$ is the number of components of the order parameter.

For $d \leqslant 3, a^{*}=a\left(T_{c}\right)=-r^{*} c>0$ and $\mathrm{P}(\eta)$ is non-Gaussian (as distinct from [28], only due to the relaxation dynamics of $\eta_{i}$ ). Keeping in mind that the softmode frequency $\Omega_{q}$ in equations (5.12), (5.28) is given by the pole of the ordinary phonon Green function $(\omega \rightarrow 0)(\mathrm{cl} .[7])$,

$$
G_{q}(\omega)=A \sum_{j} e^{\underline{i} q\left(l_{i}-l_{j}\right)}\left\langle\left\langle u_{i} \mid u_{j}\right\rangle\right\rangle_{\omega}=\left\{(m / A) \omega^{2}-\left[\Delta+f_{0}-f_{q}+M_{q}(\omega)\right]\right\}^{-1},
$$

where $M_{q}(\omega)$ is a self-energy operator, a straightforward inspection of limits $T \rightarrow$ $T_{c}^{0}, q \rightarrow 0$ yields

$$
\Omega_{q}^{2} \approx \Omega_{0}^{2}+c^{2} q^{2} ; \quad \Omega_{0}^{2}=\Delta+M_{0}(0) .
$$

Thus, while in the homogeneous case, as usual $\Omega_{0}\left(T \rightarrow T_{c}^{0}\right) \rightarrow 0$, for $d \leqslant 3-$ on account of nonlinear effects (equations (5.16), (5.37)) - one finds $\Omega_{0}^{2}\left(T \rightarrow T_{c}\right) \rightarrow$ $2\left\langle\eta^{2}\right\rangle_{c}=2 a \rightarrow-2 r^{*} c>0, T_{c}<T_{c}^{0}$. As a consequence, when $T \rightarrow T_{c}$ the displacive phase transition turns to be of the order-disorder (Ising) type governed by the relaxation cluster dynamics, i.e. $\left\langle\eta_{i}\right\rangle_{c}=0$, but $\left\langle\eta_{i}^{2}\right\rangle_{c} \sim-r^{*} c \neq 0$, and a complete phonon softening, i.e. a long-homogenous order $\left(\eta_{i}=\eta \equiv \overline{\left\langle\eta_{i}\right\rangle}=0\right)$, fails to occur.

To outline the critical cluster dynamics we use the phenomenological equation of Landau-Khalatnikov in the coordinate space (see, e.g., [32]),

$$
\partial_{\tau} \eta-\nabla^{2} \eta+r \eta+4 g \eta^{3}=0,
$$

where $\tau=\Gamma t, \Gamma$ is a "kinetic" phenomenological parameter. For $d=1$ this equation has a soliton solution: $\eta=f(x-v \tau, \tau)$ in the form $(|v| \gg 1$ or $|\triangle \eta| \ll 1 ; \zeta=x-v \tau)$

$$
f^{2}(\zeta, \tau)=-r /[4 g+\exp (-2 \zeta \tau / v)]
$$

which describes the destruction of clusters arising as fluctuations around $T_{c}$.

The spectrum of $\eta_{q}$-fluctuations in the "harmonic" approximation (if $g=0$ ) is given by [33]:

$$
I_{q}(\omega)=\int_{-\infty}^{+\infty} \mathrm{d} t e^{-\underline{i} \omega t}\left\langle\eta_{q}(0) \eta_{-q}(t)\right\rangle=\frac{k_{\mathrm{B}} T}{r+q^{2}} \frac{2 \tau_{q}}{1+\left(\omega \tau_{q}\right)^{2}}=\frac{2 \Gamma\left(k_{B} T\right)}{\tau_{q}^{-2}+\omega^{2}},
$$

where the correlation time,

$$
\tau_{q}=\left[\Gamma\left(r+q^{2}\right)\right]^{-1} ; \quad r \sim a \sim\left(T-T_{c}^{0}\right),
$$

and the reduced wave vector $q$ are introduced. 
By performing calculations for nonlinear effects $(g \neq 0)$, using ansatz [32], the asymptotic spectral response (i.e. when $q \rightarrow 0, \omega \ll 1, T \rightarrow T_{c}$ ) exhibits a central peak behaviour, thus reflecting a slow relaxation of cluster walls:

$$
I(q=0, \omega) \sim \frac{k_{\mathrm{B}} T_{c}}{\pi} \frac{\Gamma}{\omega^{1+(2-\eta) / \Delta \omega}} .
$$

The combination of the small correlation critical $(\eta)$ and dynamical $\left(\Delta_{\omega}\right)$ indices in equation (5.44) is given by the fixed point-parameter $\left(g^{*}\right)$, i.e. by the $\varepsilon$-expansion (cf. [6,32] and Refs. therein):

$$
\frac{2-\eta}{\Delta_{\omega}}=1-\frac{\left(g^{*}\right)^{2}}{8 \pi^{4}} 9 \ln (4 / 3) \approx 1-\frac{\varepsilon^{2}}{2(n+8)} \ln (4 / 3) .
$$

To conclude, our theoretical findings based on the decomposing picture on "slow" and "fast" dynamical variables (equation (5.2)) show that (in accord with the previous results, i.e. with various above mentioned approaches and suitable experiments (cf. $[7,11])$ ) at some ("true") critical temperature, $T_{c}=T_{c}^{0}-\delta T_{c}$, $\delta T_{c} \sim \varepsilon T_{c}^{0}$, the traditionally displacive system becomes structurally unstable against nonlinearly induced precursor clusters (i.e. by their order-disorder described by Ising-like variables $\left.\eta_{i}\right)$, so that $\left\langle\eta_{i}\left(T \rightarrow T_{c}\right)\right\rangle \rightarrow 0$ without a complete phonon softening: $\Omega_{q}\left(q \rightarrow 0, T \rightarrow T_{c}\right) \rightarrow 2\left\{\eta_{i}^{2}\left(T_{c}\right)=2 a^{*} \sim \varepsilon\right.$. These clusters relax in a peculiar (dynamical) fashion: $I_{q}\left(\omega \rightarrow 0, q \rightarrow 0, T \rightarrow T_{c}\right) \sim \omega^{-\lambda}, \lambda \lesssim 2$.

It should be gratifying that the present description can, at least in part, contribute to a better understanding of the SPT-dynamics, and to corroborate the appealing idea about the intrinsical "order-disorder origin" of universality.

\subsection{The pseudospin dynamics: the limiting order-disorder transition and universal features of SPT}

So far in the unified model description of SPT, i.e. in the corresponding selfconsistent procedure exposed in the preceding sections, the dynamics of a pseudospin subsystem as described by the transverse Ising model (equation (4.6)) has not been taken into account unless intermediary through the average pseudospin components $\sigma^{x}$ and $\sigma^{z}$ (equation (4.21)) in the mean-field approximation. In the case of the order-disorder SPT the soft-phonon picture of critical dynamics has to be replaced by the concept of a tunnelling mode to a great extent resembling the phonon soft mode as the instability (critical) temperature is approached. Consequently, to be properly considered, the searching for the true, universal nature of the SPT, i.e. the real physical systems undergoing structural transitions, has to take into account, in an equal manner, all the intriguing features of critical dynamics comprising phonons, tunnelling(magnon-like) and domain-wall excitations. Thus, it has surfaced an important conclusion that the system can be considered to have not one, but three characteristic frequencies: the pseudoharmonic frequency within either side of a double well, the tunnelling frequency characterizing interwell motion and a third one corresponding to domain-wall excitations (moving 
domain walls), by which the order parameter changes from one potential minimum to another inside the well defined distance [34,35]. Moreover, inside these slow moving clusters the particles participate in a quantum-mechanical flippings between their momentary rest positions $r_{i}(t)$ (equation (5.3)) which are described by the pseudospin (i.e. transverse Ising) Hamiltonian (4.6).

Within the framework of a classical-quantum approach proposed recently [35], the pseudospin dynamics (as a counterpart of the displacive type dynamics) can be treated in an almost analogous way. In the spirit of the preceding approach and definitions (section 4 and subsection 5.6), as well as introducing the representation of decomposed variables of the form (5.2), the pseudospin components can be represented as follows:

$$
S_{i}^{\alpha}=\sigma_{i}^{\alpha}+\chi_{i}^{\alpha}
$$

$$
\begin{aligned}
\sigma_{i}^{x} & =\left\langle S_{i}^{\zeta}\right\rangle \sin \theta_{i} \cos \varphi_{i} ; \chi_{i}^{x}=\delta S_{i}^{\zeta} \sin \theta_{i} \cos \varphi_{i}+S_{i}^{\xi} \cos \theta_{i} \cos \varphi_{i}-S_{i}^{\eta} \sin \varphi_{i}, \\
\sigma_{i}^{y} & =\left\langle S_{i}^{\zeta}\right\rangle \sin \theta_{i} \sin \varphi_{i} ; \chi_{i}^{y}=\delta S_{i}^{\zeta} \sin \theta_{i} \sin \varphi_{i}+S_{i}^{\xi} \cos \theta_{i} \sin \varphi_{i}+S_{i}^{\eta} \cos \varphi_{i}, \\
\sigma_{i}^{z} & =\left\langle S_{i}^{\zeta}\right\rangle \cos \theta_{i} ; \quad \chi_{i}^{z}=\delta S_{i}^{\zeta} \cos \theta_{i}-S_{i}^{\xi} \sin \theta_{i} .
\end{aligned}
$$

Here $\varphi_{i}, \psi_{i}=0$ and $\theta_{i}$ are the Euler angles after the unitary transformation in the pseudospin space $O x y z$. Apparently, new temperature dependent axes $0 \xi \eta \zeta$ are such that the mean value $\left\langle\mathbf{S}_{i}\right\rangle_{\left\{\chi_{i}^{\alpha}\right\}}$ is directed along $0 \zeta$-axis*.

In the scheme of the local (site dependent) mean field approximation (LMFA) [35] angle $\theta_{i}$ is given by (see subsection 4.3)

$$
\sin \theta_{i}=2 \Omega / H_{i}, \quad H_{i}^{2}=(2 \Omega)^{2}+H_{i z}^{2}, \quad H_{i z}^{2} \simeq\left(4 J_{0} \sigma_{i}^{z}\right)^{2},
$$

while angle $\varphi_{i}$ is expressed via $\theta_{i}[36]$,

$$
\begin{aligned}
\cos \varphi_{i} & =\frac{1}{2 \lambda \sin \theta_{i}}\left\{\beta^{2}+\left[\left(1-\beta^{2}\right)\left(4 \lambda^{2} \sin ^{2} \theta_{i}-\beta^{2}\right)\right]^{1 / 2}\right\} \\
\lambda & =\frac{1}{2} \sin \theta_{0}
\end{aligned}
$$

where $\sin \theta_{0}$ corresponds to a monodomain at given temperature and $\beta=v / c_{0}$ is the velocity of the domain-wall (in units of its maximum value $c_{0}=2 \Omega l / \hbar \sqrt{2}, l$ the lattice constant) which evolves in the pseudospin space.

The corresponding quasisoliton solution $\sigma^{z}(x, t)$ is of the same form obtained for $\eta(x, t)(5.18)$ (by using the Lorentz transformation, $x \rightarrow y$ ) as a consequence of the equilibrium condition i.e. minimization of the free energy of the pseudospin subsystem for a given configuration $\left\{\sigma_{i}^{\alpha}\right\}$ :

*For simplicity we omitted the averaging index associated with magnon-like variables $\left\{\chi_{i}^{\alpha}\right\}$ in equation (5.47). 


$$
\frac{\partial}{\partial \sigma_{i}^{z}} F\left(\left\{\sigma_{i}^{\alpha}\right\}\right)=0 ; F\left(\left\{\sigma_{i}^{\alpha}\right\}\right)=-k_{\mathrm{B}} T \ln \operatorname{Sp}_{\left\{\chi_{i}^{\alpha}\right\}} e^{-\beta H_{I M T F}}
$$

where $H_{I M T F}$ is the pseudospin Hamiltonian (4.6).

In the critical temperature region $\left(T \sim T_{c}\right)$, in the LMFA-approximation, the above equation (5.50) acquires the same form (5.16) in which the following identifications have to be carried out [35] (cf. also [11]):

$$
\begin{aligned}
\eta(x, t) & \equiv 2 \sigma^{z}(x, t) ; A \equiv 2\left[\frac{\beta_{c} \Omega}{\sinh \left(\beta_{c} \Omega\right)}\right]^{2} k_{B} T_{c}, B \equiv \frac{J_{0}^{3}}{\Omega^{2}}\left[1-\frac{\beta_{c} J_{0}}{\cosh ^{2}\left(\beta_{c} \Omega\right)}\right] \\
\beta_{c} & =1 / k_{B} T_{c} ; T_{c} \sim J_{0} .
\end{aligned}
$$

By introducing the quantum (flipping) effects in the effective (average-crystal) lattice (the bar symbol being associated with such an approximation)-through the boson-like representation,

$$
\delta S_{i}^{\zeta}=-a_{i}^{+} a_{i}^{-}, \quad S_{i}^{ \pm}=\sqrt{2 \overline{\left\langle S_{i}^{\zeta \zeta}\right.}} a_{i}^{\mp},
$$

or by directly using the adopted pseudospin Green function $[7,19]$,

$$
G_{q}^{\xi \xi}(\omega)=-\frac{\bar{H} \overline{\left\langle S^{\zeta}\right\rangle}}{\omega^{2}-\omega_{q}^{2}},
$$

the frequency of the pseudospin (tunnelling) mode is expressed in the well-known but slightly modified form [7,11,33-35];

$$
{\overline{\omega_{q}}}^{2}=\overline{H^{2}}\left(1-J_{q} \overline{\sin ^{2} \theta} / J_{0}\right) ; \quad \overline{H^{2}}=(2 \Omega)^{2}+\left(4 J_{0}\right)^{2}\left\langle\left(\sigma^{z}(x)\right)^{2}\right\rangle_{c} .
$$

It is easily seen that the results analogous to the case of displacive SPT (elaborated in the previous subsection) are obtained: with the onset of criticality an incomplete softening of the pseudospin (tunnelling) mode occurs,

$$
\bar{\omega}^{2}\left(q \rightarrow 0, T \rightarrow T_{c}\right) \simeq\left(4 J_{0}\right)^{2}\left\langle\left(\sigma^{z}\right)^{2}\right\rangle_{c} \neq 0
$$

while in the spectral density of excitations a central peak of the form (5.44),

$$
S(q=0, \omega) \sim \omega^{-\lambda}, \quad \lambda \lesssim 2,
$$

evolves as a consequence of the pseudospin domain-wall relaxation.

Instead of extended conclusions usually presented at the end of each section, it is gratifying that the present contribution can, at least in part, complete a better understanding of the SPT dynamics and to corroborate, the more and more appealing idea about the intrinsical order-disorder nature of SPT as its essential universal feature. Although invalid in quantitative predictions, our unified model 
description of SPTs (bearing in mind simple approximations used) is illuminating, as Drs. Bruce and Cowley, great specialists in this field, stated in [11].

We hope that the theoretical results presented in this review have "displaced" and contributed to the "order" of some aspects of knowledge about the universal nature of SPTs, as well as to a prompt further progress in all-inclusive SPT investigations.

\section{Acknowledgement}

I would like to gratify Prof. Academician I.R.Yukhnovskii for his kind invitation to participate in the special issue of the journal Condensed Matter Physics devoted to the 60th anniversary of Professor, Dr. Ihor Stasyuk, my dear colleague and an outstanding scientist. It was my great pleasure that I had an opportunity to cooperate with Dr. Ihor Stasyuk during all these years. I would like to express my wishes of all the best in his future scientific work and his life.

\section{References}

1. Stamenković S., Plakida N.M., Aksienov V.L., Siklós T., Phys. Rev B, 1976, vol 14, p. 5080; JINR, P17-9226, Dubna, 1975 (in Russian).

2. Stamenković S., Plakida N.M., Aksienov V.L., Siklós T., Ferroelectrics, 1976, vol 14, p. 655 .

3. Stamenković S., Plakida N.M., Aksienov V.L., Siklós T., Acta Phys. Hung., 1977, vol 42, p. 265.

4. Stamenković S., Plakida N.M., Aksienov V.L., Siklós T., Acta Phys. Hung., 1977, vol 43, p. 99.

5. Stamenković S.,Plakida N.M., Aksienov V.L., Siklós T., Fizika, 1978, vol 10, Supplement 2, p. 122; Stamenković S., Plakida N.M., Aksienov V.L., Siklós T., Ferroelectrics, 1980, vol 24, p. 255; JINR, P17-11856, Dubna, 1978 (in Russian);Aksenov V.L., D. Baatar, N.M. Plakida, S. Stamenković, JINR, P17-12961, Dubna, 1979 (in Russian).

6. Stamenković S., Plakida N.M, Aksienov V.L., Zagrebnov V.A., Fizika, 1980, vol 12, Supplement 1, p. 332; Aksenov V.L., Zagrebnov V.A., Plakida N.M., Stamenković S., JINR, P17-80-576, Dubna, 1980 (in Russian).

7. Aksienov V.L., Plakida N.M., Stamenković S., Neutron scattering by Ferroelectrics, World Scientific, Singapore, 1989.

8. Plakida N.M, in Statistical Physics and Quantum Theory of Fields, 1973, ed. Bogolyubov N.N., Nauka, Moscow, p. 205-240 (In Russian).

9. Fujiwara T., J. Phys. Soc. Japan, 1970, vol 29, p. 1282.

10. Moore M.A., Williams H.C.W.L., J. Phys. C, 1972, vol 5, p. 3168.

11. Bruce A.D, Cowley R.A. Structural Phase Transition, Taylor and Francis Ltd., London, 1981.

12. Vaks V.G., Larkin A.I., Zhurn. Eksp. Teor. Fiz., 1965, vol 49, p.975 (in Russian).

13. Plakida N.M, Siklós T., Acta Phys. Hung., 1978, vol 45, p. 37.

14. Aksienov V.L., Breter H., Kovalski Ya.M., Plakida N.M., Priezzhev V.B., Fiz.tverd. tela, 1976, vol 18, p. 2920 (in Russian). 
15. Gillis N.S. and Koehler T.R., Phys.Rev. Lett., 1972, vol 29, p. 369; Phys.Rev. B, 1973, vol 7, p. 4980; Phys.Rev., 1974, vol 9, p. 3806; Gillis N.S., Phys. Rev. B, 1975, vol 11, p. 309; Gillis N.S., Dynamical Properties of Solids, vol 2, ed. G.K. Horton and A.A. Maradudin, North-Holland, Amsterdam, 1975, p. 107.

16. Eisenriegler E., Phys.Rev. B, 1974, vol 9, p. 1029.

17. Sokolov A.I., Fiz.Tverd. Tela, 1974, vol 16, p. 733 (in Russian).

18. Elliot R.G., Krumhansl J.A., Leath P.L., Rev. Mod. Phys., 1974, vol 46, p.5080.

19. Aksenov V.L., Schreiber J., Phys. Lett., 1978, vol 69A, p.56.

20. Stasyuk I.V., Preprint ITF - 75-108P, 1975, Kiev (in Russian).

21. Krumhansl J.A., Schrieffer J.R., Phys.Rev. B, 1975, vol 11, p.3535.

22. Koehler T.R., Bishop A.R., Krumhansl J.A., Schrieffer J.R., Solid State Commun., 1975, vol 17, p.1515; Schneider T., Stoll E., Phys.Rev. Lett. 1976, vol 36, p.1501; Phys.Rev.B, 1976, vol 13, p.1216; Phys.Rev. B, 1978, vol 17, p.1302.

23. Beck H., J. Phys.C, 1976, vol 9, p.33.

24. Bruce A.D., Müller K.A., Berlinger W., Phys.Rev.Lett., 1979, vol 42, p.185.

25. Reiter G.F., Berlinger W., Müller K.A., Phys.Rev. B, 1980, vol 21, p.1.

26. Müller K.A., Lecture Notes in Physics, ed. Enz C.P., Springer-Verlag, Berlin, 1979, p. 210.

27. Bruce A.D., Schneider T., Phys.Rev. B, 1977, vol 16, p. 3991.

28. Bruce A.D., Schneider T., Stoll E., Phys. Rev. Lett., 1979, vol 43, p. 1284.

29. Beale P.D., Sarker S., Krumhansl J.A., Phys.Rev. B, 1981, vol 24, p. 266.

30. Bishop A.R., Domany E., Krumhansl J.A., Phys.Rev., 1976, vol 14, p. 2966.

31. Aksenov V.L., Didyk A.Yu., Yushankhaj V.Yu, Solid State Commun., 1981, vol 40, p. 825; Fiz. Niz. Temp., 1982, vol. 8, p. 626 (in Russian).

32. Patashinskii A.Z., Pokrovskii V.L., Fluctuation Theory of Phase Transitions, Nauka, Moscow, 1975, vol. 5, p.211 (in Russian); ibid, II edition, Nauka, Moscow 1982, p. 277 (in Russian).

33. Blinc R, Žekš B., Soft Modes in Ferroelectrics and Antiferroelectrics, North-Holland, Amsterdam 1974, p. 54.

34. Lines M.E. and Glass A.M., Principles and Applications of Ferroelectrics and Related Materials, Clarendon Press, Oxford, 1977, Sections 1 and 2.

35. Stamenković S., Simp. Fiz. Kondenz. Materije, ed. M. Davidović, Kladovo, 1997, p. 129 (in Serbian).

36. Prelovšek P., Sega I., J. Phys. C: Solid State Phys. 1981, vol. 14, 3231. 


\section{Уніфікований модельний опис структурних фазових переходів типу порядок-безпорядок та типу зміщення}

\section{С.Стаменкович}

Інститут ядерних наук, Лабораторія теоретичної фізики та фізики конденсованої речовини, Бєлград, Пошт. Скр. 522, Югославія

Отримано 7 липня 1998 р.

Зроблено огляд і часткове оновлення серії робіт автора з співавторами [1-7], присвяченої уніфікованому модельному опису структурних фазових переходів (СФП) у сегнетоелектриках та споріднених матеріалах.

Виходячи з загального гамільтоніану попарно зв'язаних ангармонічних (четвірних) осциляторів та враховуючи концепцію локальних нормальних координат, запропоновано уніфікований модельний опис систем з СФП обидвох типів: порядок-безпорядок і зміщення.

В рамках стандартної варіаційної процедури формулюється гібридизований псевдоспін-фононний гамільтоніан введенням змінних, що відповідають фононним, магноно-подібним (з переворотом) і нелінійним (типу доменної стінки) зміщенням атомів, що беруть участь у СФП. Це досягається представленням колективного руху атомів через декілька квазірівноважних положень (в найпростішому випадку - двох) як повільного тунельного зміщення (розбитого на магноноподібні і солітоно-подібні відхилення) додатково до порівняно швидких фононних осциляцій навколо неоднорідних миттєвих положень спокою, в свою чергу індукованих збудженнями типу доменної стінки (солітонами).

Якісний і кількісний аналізи показали, що СФП (першого чи другого роду) можуть бути типу зміщення (керованих м'якою фононною модою), порядок-безпорядок (керованих тунельною магноноподібною м'якою модою) чи змішаного типу залежно як від енергії зв'язку між атомами, так і від їхньої нульової точки коливної енергії. В області критичної температури збудження типу доменної стінки приводять до утворення мікродоменів (кластерів впорядкованої фази), які індукують СФП класу універсальності Ізінгівського типу. Має місце неповне пом'якшення фононної чи псевдомагнонної моди і у спектральній густині збуджень виникає центральний пік, викликаний повільною релаксацією кластерів.

Ключові слова: структурні фазові переходи, порядок-безпорядок, перехід зміщення

PACS: $63.70 .+h, 77.80 . B h, 64.60 .-i, 64.60 . C n$ 
